ARTICLE

\title{
Synthetic essentiality between PTEN and core dependency factor PAX7 dictates rhabdomyosarcoma identity
}

Casey G. Langdon', Katherine E. Gadek (1) 1, Matthew R. Garcia', Myron K. Evans (1) 1, Kristin B. Reed ${ }^{1,2}$, Madeline Bush ${ }^{1,3}$, Jason A. Hanna ${ }^{1,7}$, Catherine J. Drummond ${ }^{1,8}$, Matthew C. Maguire ${ }^{1}$, Patrick J. Leavey (1) ${ }^{1}$, David Finkelstein ${ }^{4}$, Hongjian Jin ${ }^{5}$, Patrick A. Schreiner ${ }^{5}$, Jerold E. Rehg ${ }^{6}$ \& Mark E. Hatley (D) ${ }^{1 凶}$

PTEN promoter hypermethylation is nearly universal and PTEN copy number loss occurs in $\sim 25 \%$ of fusion-negative rhabdomyosarcoma (FN-RMS). Here we show Pten deletion in a mouse model of FN-RMS results in less differentiated tumors more closely resembling human embryonal RMS. PTEN loss activated the PI3K pathway but did not increase mTOR activity. In wild-type tumors, PTEN was expressed in the nucleus suggesting loss of nuclear PTEN functions could account for these phenotypes. Pten deleted tumors had increased expression of transcription factors important in neural and skeletal muscle development including $D b x 1$ and Pax7. Pax7 deletion completely rescued the effects of Pten loss. Strikingly, these Pten;Pax7 deleted tumors were no longer FN-RMS but displayed smooth muscle differentiation similar to leiomyosarcoma. These data highlight how Pten loss in FN-RMS is connected to a PAX7 lineage-specific transcriptional output that creates a dependency or synthetic essentiality on the transcription factor PAX7 to maintain tumor identity.

\footnotetext{
${ }^{1}$ Department of Oncology, St. Jude Children's Research Hospital, Memphis, TN 38105, USA. ${ }^{2}$ Rhodes College, Memphis, TN 38112, USA. 3 St. Jude Graduate School of Biomedical Sciences, Memphis, TN 38105, USA. ${ }^{4}$ Department of Computational Biology, St. Jude Children's Research Hospital, Memphis, TN 38105, USA. ${ }^{5}$ Center for Applied Bioinformatics, St. Jude Children's Research Hospital, Memphis, TN 38105, USA. ${ }^{6}$ Department of Pathology, St. Jude Children's Research Hospital, Memphis, TN 38105, USA. 'Present address: Purdue Center for Cancer Research, Department of Biological Sciences, Purdue University, West Lafayette, IN, USA. ${ }^{8}$ Present address: Department of Pathology, University of Otago, Dunedin, Otago, New Zealand.

凶email: mark.hatley@stjude.org
} 
R habdomyosarcoma (RMS) is the most common soft-tissue sarcoma in childhood and high-risk RMS patients have a 5 -year survival rate of only $20 \% 1$. RMS is classified into two major histologic subgroups, alveolar (ARMS) and embryonal $(\text { ERMS })^{2}$. The majority of ARMS tumors ( $\left.80 \%\right)$ harbor chromosomal translocations resulting in expression of either PAX3FOXO1 or PAX7-FOXO1 oncofusion proteins which foretell a worse prognosis ${ }^{1}$. ARMS tumors without oncofusion proteins both molecularly and clinically resemble ERMS, thus classification as either fusion-positive (FP-RMS) or fusion-negative (FNRMS) better reflects the biological and clinical features of $\mathrm{RMS}^{1}$. FN-RMS tumors are genetically heterogeneous with a constellation of putative driver mutations and loss-of-function alterations in tumor suppressors such as TP53 and CDKN2A; the majority of tumors have no apparent driver mutations ${ }^{3-7}$. In contrast, hypermethylation of the Phosphatase and Tensin Homolog $(P T E N)$ promoter and decreased expression was found in over $90 \%$ of FN-RMS tumors ${ }^{6}$. PTEN promoter hypermethylation is one of many ways that PTEN expression can be modulated in cancer ${ }^{8,9}$. Furthermore, a subset of FN-RMS tumors also harbors PTEN mutations ${ }^{10}$.

PTEN's tumor-suppressive function is thought to be secondary to dephosphorylation and inactivation of the lipid second messenger phosphatidylinositol-3,4,5-triphosphosphate, a potent $\mathrm{PI} 3 \mathrm{~K} / \mathrm{AKT} / \mathrm{mTOR}$ pathway activator ${ }^{11-13}$. Inhibition of the $\mathrm{PI} 3 \mathrm{~K} / \mathrm{AKT} / \mathrm{mTOR}$ pathway is an attractive RMS therapy but has yielded varied results ${ }^{3,14-16}$. However, increasing evidence suggests PTEN maintains a myriad of tumor-suppressive functions aside from negatively regulating the PI3K pathway ${ }^{11}$. PTENdeficient tumors require the expression of other genes to maintain their proliferative state; these synthetic essential gene interactions create therapeutically exploitable gene dependencies ${ }^{17,18}$. Using an integrative expression and copy number computational approach, PTEN was suggested to be a key tumor suppressor in FN-RMS ${ }^{19}$. However, PTEN's biological function in FN-RMS is largely unexplored. Herein, we leverage a Hedgehog-driven, FNRMS mouse model to interrogate the role of PTEN in FN-RMS biology ${ }^{20,21}$. In contrast to modulating the PI3K/AKT/mTOR pathway, we reveal PTEN loss drives a transcriptional axis centered on PAX7 necessary for FN-RMS tumor identity.

\section{Results}

Pten loss exacerbates aP2-Cre;SmoM2 FN-RMS development. The nearly universal PTEN promoter hypermethylation in FN$\mathrm{RMS}^{6}$ led us to investigate whether Pten loss is sufficient to drive rhabdomyosarcomagenesis by conditionally deleting a Pten flox allele with Cre recombinase expressed from the adipose protein 2 (aP2) promoter (Supplementary Fig. 1a) ${ }^{22}$. Neither aP2-Cre;Ptenflox/flox (APcKO) nor aP2-Cre;Ptenflox/+ (APcHet) compound mutant mice developed tumors. APcKO mice had significantly larger intrascapular brown adipose tissue (BAT) but not inguinal white adipose tissue (iWAT) than APcHet littermates (Supplementary Fig. 1b-d). Histologically, the BAT was hyperplastic without evidence of hibernoma (Supplementary Fig. 1e). The iWAT displayed hyperplastic cellularity without any overt pathology (Supplementary Fig. 1f). Thus, Pten loss is not sufficient to drive rhabdomyosarcomagenesis in aP2-Cre mice.

In order to understand the consequences of PTEN loss in FN-RMS, we sought to conditionally delete Pten flox in our murine FN-RMS model resulting from activation of a conditional, constitutively active Smoothened allele (SmoM2) by aP2-Cre ${ }^{20,21}$. We bred aP2-Cre;Pten flox/+ mice to Smo ${ }^{M 2 / M 2} ;$ Pten $^{\text {flox } /+}$ mice to generate aP2-Cre;Smo ${ }^{\mathrm{M} 2 /{ }_{+}}$;Pten flox/flox (ASPcKO), aP2-Cre;S$\mathrm{mo}^{\mathrm{M} 2 /+}$;Ptenflox/ $\quad$ (ASPcHet), and aP2-Cre;Smo ${ }^{\mathrm{M} 2 /+}{ }_{;}$Pten $^{+/+}$ $(\mathrm{ASP} T \mathrm{~T})$ compound mutant mice (Fig. 1a). Pten loss accelerated tumor formation with a median tumor-free survival of 9 days in $\mathrm{ASP}^{\mathrm{CKO}}$ mice compared to 16 days in ASPWT mice (Fig. $1 \mathrm{~b}$ and Supplementary Fig. 2a). All tumors in ASPcKO mice occurred in the head and neck without any evidence of regional lymph node or pulmonary metastasis at necropsy just as in the ASPWT mice. In addition, ASPcKO mice had increased tumor burden and decreased body weight compared to ASPWT mice (Fig. 1c and Supplementary Fig. 2b-d).

Histologic examination revealed ASPcKO tumors contained malignant proliferations with vague, haphazard spindle cell growth patterns comprised of cohesive cells with coalescing pale pink cytoplasm, with or without striations and irregular margins infiltrating adjacent tissue (Fig. 1d). The cells contained variably sized round, oval, or spindle nuclei. By immunohistochemistry (IHC), ASPcKO tumors strongly expressed muscle-specific intermediate filament DESMIN and nuclear staining for skeletal muscle-specific transcription factors MYOD1 and MYOGENIN (Fig. 1d and Supplementary Fig. 2e, f). In addition, ASPcKO tumors have a higher proliferative index than ASPWT tumors (Fig. 1e, f). The infiltrative pattern and cytological morphology of the small cells in ASPcKO tumors are consistent with an embryonal-like RMS rather than the well-differentiated RMS more typical of the ASPWT tumors. Importantly, PTEN protein expression by IHC showed the absence of PTEN staining in ASPcKO tumors, whereas both cytoplasmic and nuclear PTEN staining was observed in ASPWT tumors (Fig. 1g). These histologic observations suggest PTEN loss may have a functional role in maintaining a less differentiated cell state.

ASPWT tumors can be serially transplanted in NSG but not in SCID/Beige immunocompromised mice (Supplementary Fig. 2g). To test whether tumors from ASPcKO mice are more tumorigenic than $\mathrm{ASP} \mathrm{WT}^{\mathrm{T}}$ mice, we surgically implanted tumors into the flanks of SCID/Beige mice. All ASPcKO tumors implanted developed tumors that were histologically similar to FN-RMS suggesting $\mathrm{ASP}^{\mathrm{cKO}}$ tumors are more malignant than ASPWT tumors (Fig. 1h, i). The shorter latency, increased tumor burden, and enhanced tumorigenicity illustrates $\mathrm{ASP}{ }^{\mathrm{cKO}}$ tumors represent a more robust model of FN-RMS.

To determine if the effects of Pten loss were not solely reflective of generic tumor suppressor loss, we conditionally deleted Trp53, $C d k n 2 a$, and $R b 1$ independently in our aP2-Cre;SmoM2 model. The deletion of $\operatorname{Trp53}, C d k n 2 a$ or $R b 1$ in our model did not alter the tumor latency, histology, or tumor burden despite increasing the penetrance of tumor formation (Supplementary Fig. $2 \mathrm{~h}-\mathrm{q}$ ). While all ASPcKO primary tumors formed secondary tumors as allografts in SCID/Beige mice, only two of six aP2-Cre;Smo ${ }^{\mathrm{M} 2 /+}$; Trp53flox/flox and two of eight aP2-Cre;Smo ${ }^{M 2 /+} ; R b 1^{\text {flox/flox }}$ formed secondary tumors while no tumors formed in aP2-

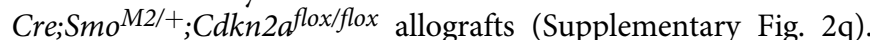
These data suggest that Pten loss provides a significant advantage to the RMS tumor cell.

PTEN loss enhances myogenic differentiation impairment in ASPcKO tumors. Skeletal muscle development is a well-studied process of tissue differentiation driven by transcriptional regulation from fetal to mature adult skeletal muscle tissue ${ }^{23}$. Both $\mathrm{ASP}^{\mathrm{CKO}}$ and ASPWT tumors had increased expression of early skeletal muscle transcription factors Myod1, Myf5, and Myog compared to the mature sternocleidomastoid (SCM) skeletal muscle by real-time PCR consistent with FN-RMS (Fig. 2a). Although there were differences in Myod1 and Myog mRNA expression between $\mathrm{ASP} \mathrm{WT}^{\mathrm{T}}$ and $\mathrm{ASP} \mathrm{CKO}$ tumors, there was no change in protein expression by IHC (Fig. 1d and Supplementary Fig. 2e, f). In contrast, ASPcKO tumors had significantly lower expression of terminally differentiated skeletal muscle genes, 
a

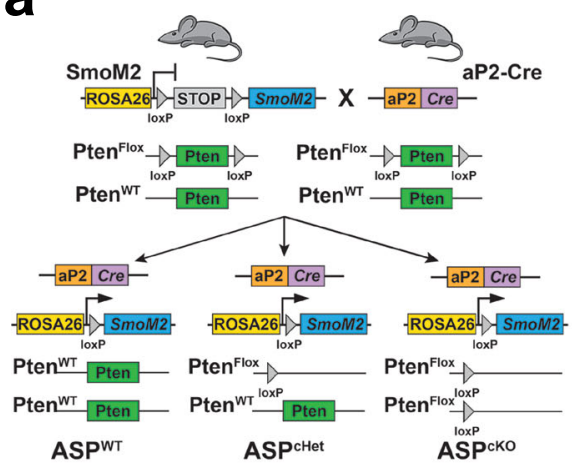

b

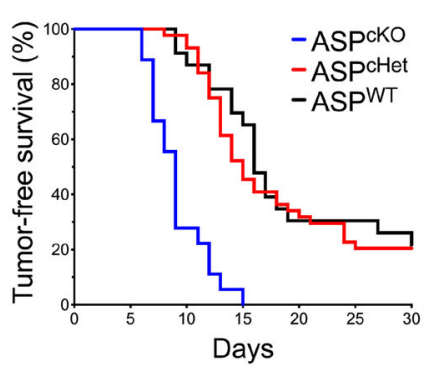

C

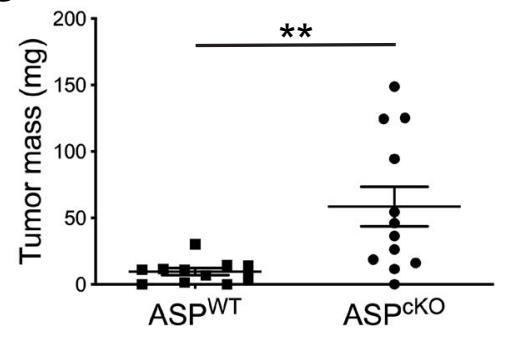

e
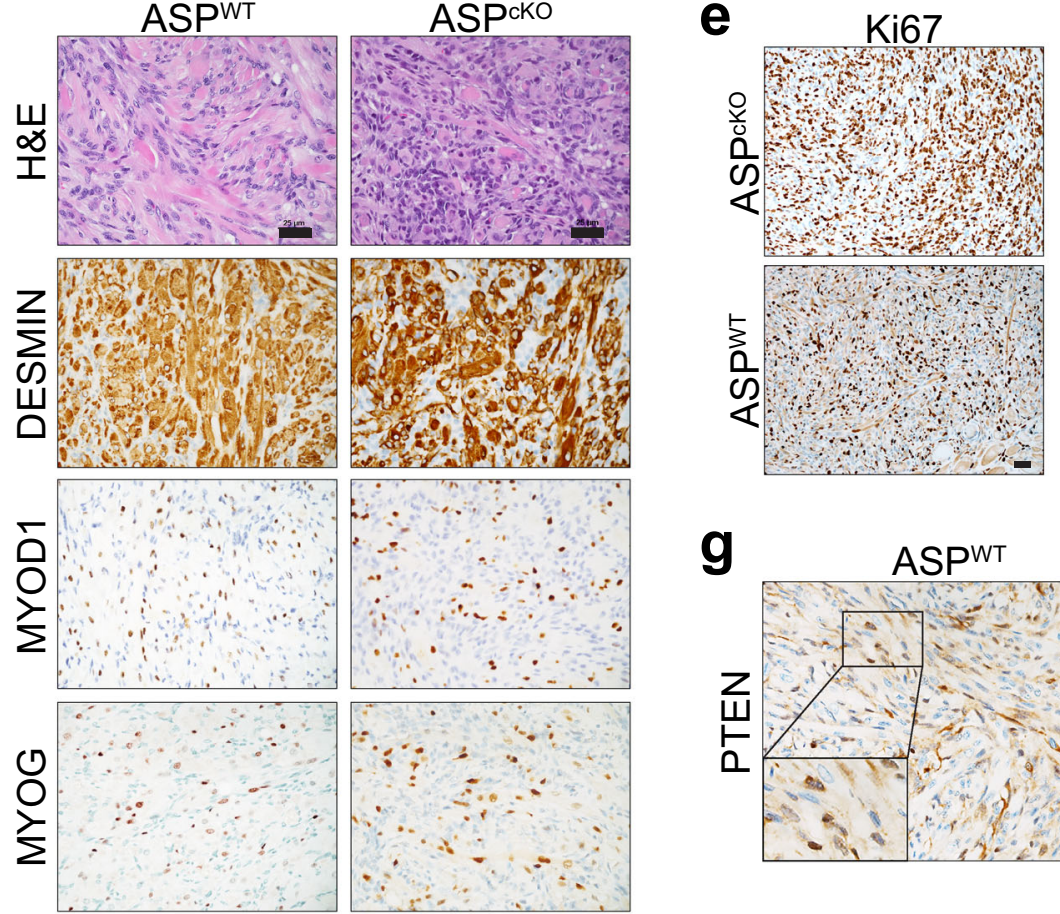

f

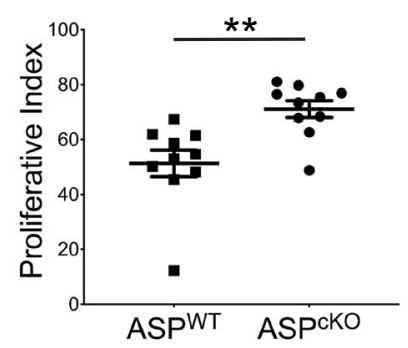

g
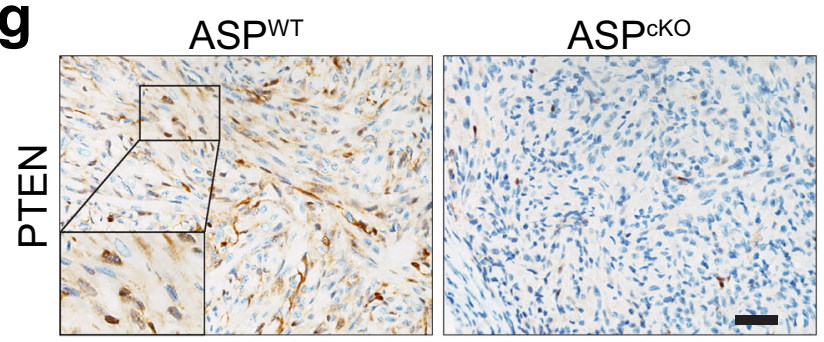

h
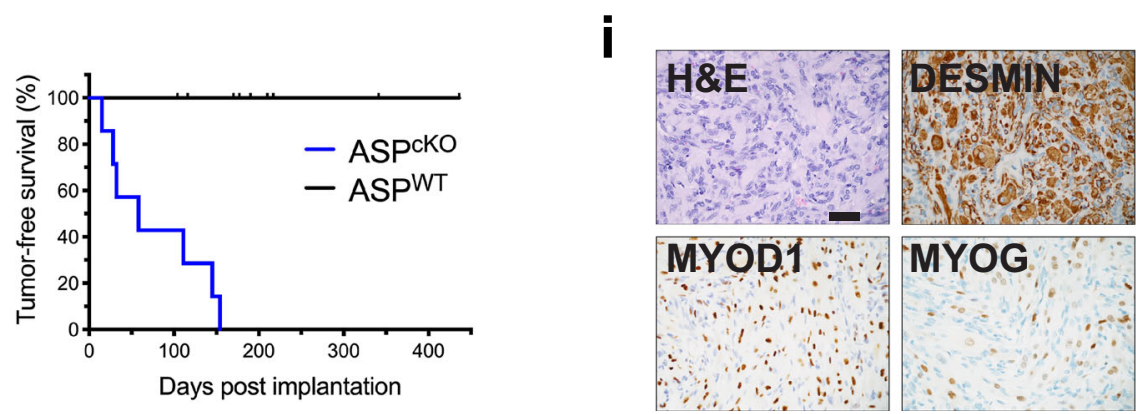

Fig. 1 Pten loss exacerbates aP2-Cre;SmoM2 FN-RMS development. a Breeding strategy to generate ASPWT, ASPcHet, and ASPCKO mice. b Kaplan-Meier tumor-free survival in ASPWT $(n=23)$, ASPcHet $(n=42)$, and ASPcKO $(n=18)$ tumors $(P<0.0001)$. c Tumor mass at P12 from ASPWT $(n=11)$ and $\operatorname{ASPCKO}(n=12)$ mice $(P=0.0052)$. d Representative histology of ASPWT $(n=16)$ and ASPcKO ( $n=16)$ tumors by H\&E and IHC staining for DESMIN, MYOD1, and MYOGENIN (MYOG). Scale bar, $25 \mu \mathrm{m}$. e, f Representative Ki67 IHC (e) and quantitation (f) in ASPWT ( $n=9$ ) and ASPCKO tumors $(n=7)$ tumors (ten random fields of view per genotype) $(P=0.0029)$. Scale bar, $25 \mu \mathrm{m}$. $\mathbf{g}$ Representative PTEN IHC in ASPWT $(n=3)$ and ASPCKO $(n=3)$ tumors. Scale bar, $25 \mu \mathrm{m}$. h Kaplan-Meier tumor-free survival curve of subcutaneous flank allografts of ASPWT $(n=11)$ and ASPCKO $(n=7)$ tumors $(P<0.0001)$. i Representative histology of ASPcKO $(n=3)$ allografts by H\&E staining and IHC for DESMIN, MYOD1, and MYOG. Scale bar, $25 \mu \mathrm{m}$. All pairwise comparisons determined by unpaired, two-tailed Student's $t$ test. Kaplan-Meier survival curves analyzed with Mantel-Cox log-rank test. ${ }^{\star \star} P<0.01$, mean \pm SEM. See also Supplementary Figs. 1 and 2. Source data are provided as a Source Data file. 
a<smiles>[AsH3]</smiles>

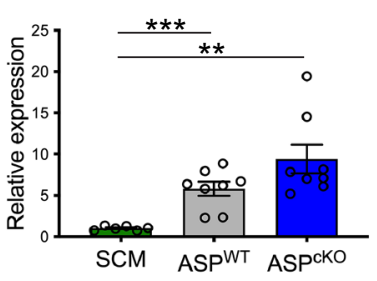

b

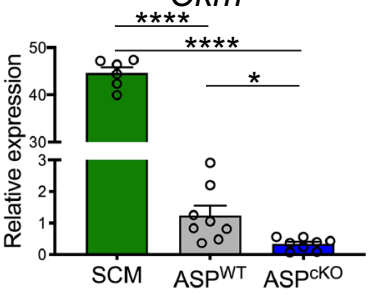

d
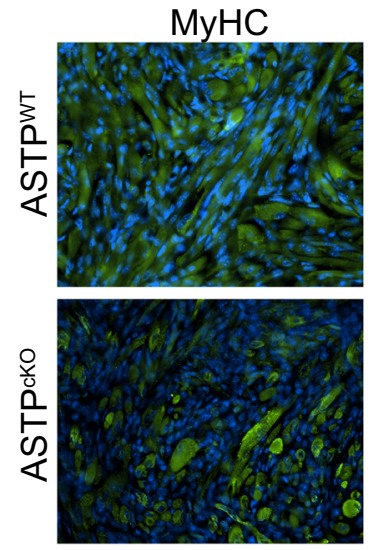

f
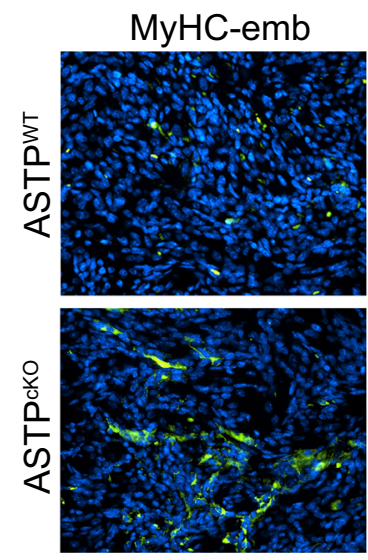
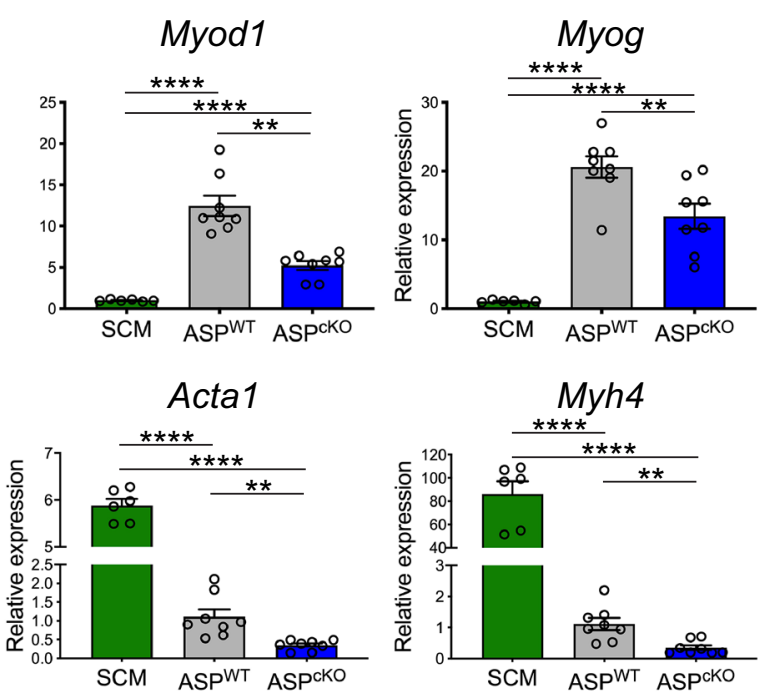

C
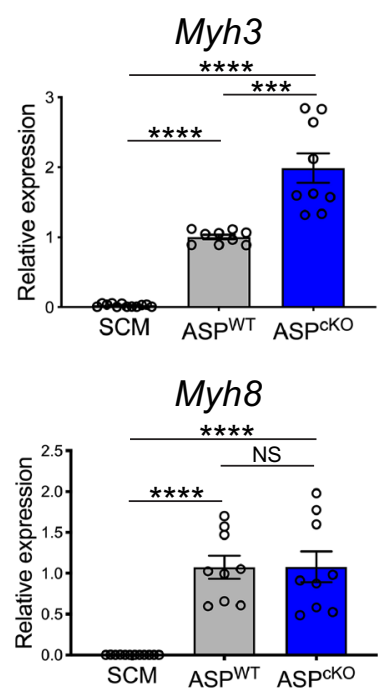

e
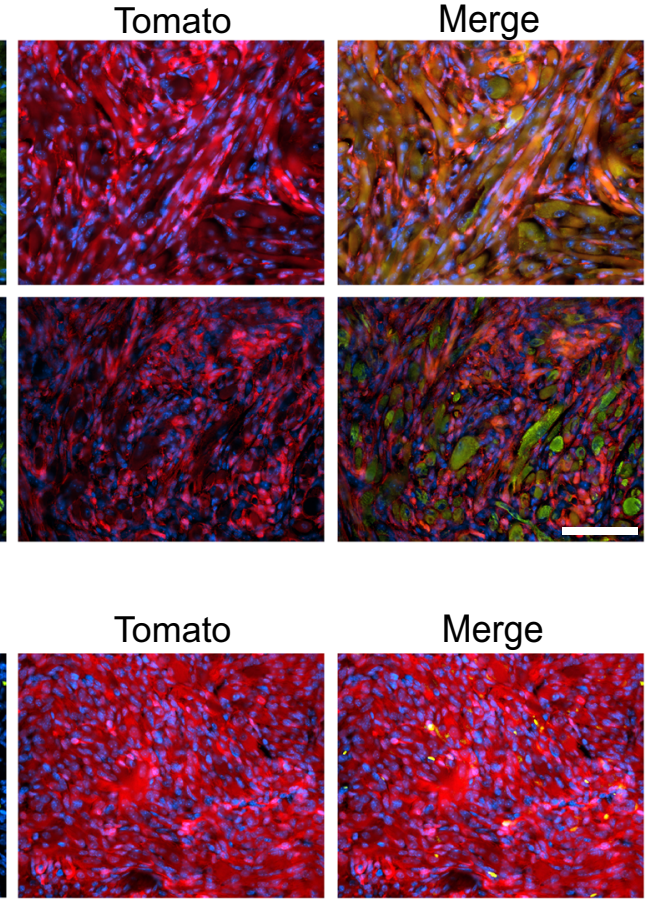

g

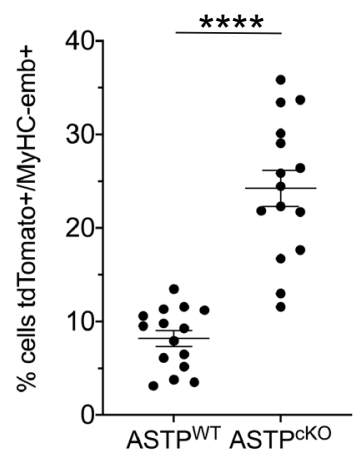

Acta1, Ckm, and Myh4 (Fig. 2b). This suggests that Pten loss further attenuates the differentiation of the skeletal myogenic program within the FN-RMS tumor cells.

Similar to the series of transcription factors driving skeletal muscle determination and differentiation, myosin heavy chain (MyHC) isoforms of sarcomeric filaments display a developmental progression from MyHC-embryonic (MYH3) to MyHCneonatal (MYH8) to finally adult MyHCs (MYH1, MYH2, MYH4 ${ }^{23}$. MyHC-embryonic encoded by Myh3 which is only expressed in embryonic developing skeletal muscle is also increased in the ASPcKO tumors compared to ASPWT. However, no change in expression with neonatally expressed $M y h 8$ suggests $\mathrm{ASP}^{\mathrm{CKO}}$ tumors resemble an earlier skeletal muscle progenitor (Fig. 2c). To better define the tumor cell differentiation state in the ASPCKO and ASPWT tumors, we bred Rosa2 $6^{\text {tdTomato }}$ reporter mice into the ASPWT and ASPcKO models to generate ASTP mice. In the ASTP mice, tumor cells that have expressed aP2-Cre are indelibly labeled with tdTomato allowing tumor cell visualization. Immunofluorescent staining illustrated that nearly all tdTomato ${ }^{+}$tumor cells in ASTPWT mice expressed adult 
Fig. 2 PTEN loss enhances myogenic differentiation impairment in ASPcKo tumors. a Gene expression by real-time PCR of Myf5, Myog, and Myod1 in sternocleidomastoid (SCM) muscles $(n=3)$, ASPWT $(n=4)$, and ASPCKO $(n=4)$ tumors, in duplicate. (Myf5 SCM-ASPWT P =0.004, SCM-ASPCKO $P=0.0014$ ) (Myod1 ASPWT-ASPCKO $P=0.0001$, SCM-ASPWT $P<0.0001$, SCM-ASPCKO $P<0.0001$ ) (Myog ASPWT-ASPCKO $P=0.0099$, SCM-ASPWT $P<0.0001$, SCM-ASPCKO $P<0.0001)$. b Gene expression by real-time PCR of Acta1, Myh4, and Ckm in SCM $(n=3), \operatorname{ASPWT}(n=4)$, and ASPcKO $(n=4)$ tumors, in duplicate. (Ckm ASPWT-ASPCKO $P=0.0136$, SCM-ASPWT $P<0.0001$, SCM-ASPCKO $P<0.0001$ ) (Acta1 ASPWT-ASPCKO $P=0.0024$, SCMASPWT $P<0.0001$, SCM-ASPCKO $P<0.0001$ ) (Myh4 ASPWT-ASPCKO $P=0.0027$, SCM-ASPWT $P<0.0001$, SCM-ASPcKO $P<0.0001$ ). c Gene expression by real-time PCR of Myh3 and Myh8, myosin heavy chains. SCM $(n=3)$, ASPWT $(n=4)$, and ASPcKO $(n=4)$ tumors, in triplicate. (Myh3 ASPWT-ASPcKO $P=0.0003$, SCM-ASPWT $P<0.0001$, SCM-ASPCKO $P<0.0001$ ) (Myh8 SCM-ASPWT $P<0.0001$, SCM-ASPCKO $P<0.0001$. d, e Representative immunofluorescence (IF) from ASTPWT and ASTPCKO tumors stained for mature myosin heavy chain (MyHC, green) with endogenous tdTomato fluorescence (red). DAPI nuclear stain (blue). Representative images shown, $n=3$ tumors per genotype. Scale bar, $100 \mu \mathrm{m}$. e Quantification of tdTomato $+/ \mathrm{MHC}+$ cells in tumors from $(\mathbf{d})(P<0.0001)$. $\mathbf{f}, \mathbf{g}$ Representative IF from ASTPWT and ASTPCKO tumors stained for embryonic myosin heavy chain (MyHC-emb, green) with endogenous tdTomato fluorescence (red). DAPI nuclear stain (blue). Representative images shown, $n=3$ tumors per genotype. Scale bar, $100 \mu \mathrm{m}$. $\mathbf{g}$ Quantification of tdTomato $+/ \mathrm{MyHC}$-emb + cells in tumors from $(\mathbf{f})(P<0.0001)$. All pairwise comparisons were determined by Student's $t$ test (unpaired, two-tailed); ${ }^{\star} P<0.05,{ }^{\star \star} P<0.01,{ }^{\star \star \star} P<0.001,{ }^{\star \star \star \star} P<0.0001$. Data represented as mean $+\mathrm{SEM}$. Real-time PCR normalized to $18 \mathrm{~S}$ rRNA and expressed relative to $\operatorname{SCM}(\mathbf{a})$ or ASPWT $(\mathbf{b}, \mathbf{c})$. See also Supplementary Fig. 3. Source data are provided as a Source Data file.

MyHC compared to only $\sim 60 \%$ of the tdTomato ${ }^{+}$tumor cells in ASTPcKO mice (Fig. 2d, e). Furthermore, triple the number of tdTomato $^{+}$tumor cells in ASP ${ }^{c K O}$ mice were positive for MyHCembryonic compared to ASPWT mice while no change was observed for MyHC-perinatal (Fig. 2f, g and Supplementary Fig. 3a, b). These results further indicate that ASP ${ }^{\mathrm{cKO}}$ tumors are less differentiated than ASPWT tumors and more reflective of human ERMS.

PTEN loss activates AKT but not mTORC1 in ASPcKO tumors. Next, we sought to determine how PTEN loss affects cell signaling in the ASPCKO tumors. PTEN loss and unchecked PI3K-pathway activation lead to aberrant activation/phosphorylation of kinases important in maintaining many hallmarks of cancer ${ }^{11}$. To determine the signaling pathways regulated by PTEN loss in ASPcKO tumors, we performed an unbiased analysis of signaling proteins using reverse-phase protein arrays with 350 different antibodies directed against major cancer-related proteins. In ASPcKO tumors, 30 probes were relatively overexpressed and 25 probes were relatively underexpressed (Fig. 3a). As expected, PTEN was the protein most decreased in ASP ${ }^{\mathrm{cKO}}$ compared to ASPWT tumors with concomitant increased AKT phosphorylation at both Thr308 and Ser473 (Fig. 3a, b). Among other proteins within the AKT/mTOR pathway, only phosphorylated PRAS40 Thr246 was significantly relatively overexpressed in the ASPcKO tumors. Surprisingly, the mTOR-downstream target phosphorylated $\mathrm{p} 70 \mathrm{~S} 6 \mathrm{~K}^{\mathrm{Th} 389}$ was significantly decreased in ASPcKO tumors, suggesting that AKT phosphorylation was largely uncoupled from mTORC1, further evidenced by lack of increased phosphorylation of S6 or 4E-BP1 (Fig. 3a, b). The other proteins increased in ASP ${ }^{\mathrm{CKO}}$ tumors included both NOTCH1 and NOTCH3, constituents of MAPK cascades, and phosphorylated ERBB3 with its cognate ligand HEREGULIN. We confirmed increased ERK1/2 phosphorylation in ASPcKO bulk tumor lysates (Supplementary Fig. 4a). However, we saw no increased sensitivity to trametinib in ASP ${ }^{\mathrm{CKO}}$ primary rhabdospheres; this was probably due to a lack of maintained ERK1/2 phosphorylation observed in cultured ASP ${ }^{\mathrm{CKO}}$ primary rhabdospheres (Supplementary Fig. 4b, c). Furthermore, immunosuppressive molecules B7-H3, CD86, and CD38 as well as necroptosome components cleaved caspase- 8 and RIP3 (also known as RIPK3) were relatively underexpressed in ASP ${ }^{\mathrm{CKO}}$ tumors suggesting less immune surveillance in the tumor microenvironment with Pten loss.

Given the increased AKT phosphorylation and absence of concomitant increased mTOR signaling in the ASP ${ }^{\mathrm{cKO}}$ tumors, we examined whether these signaling results correlated with increased sensitivity to PI3K, mTOR, or AKT inhibitors. We dissected and dissociated ASPWT and ASP ${ }^{c K O}$ tumors, seeded dissociated tumor cells to grow as rhabdospheres, and assayed cell growth when incubated with either GDC-0941 (pan-PI3K inhibitor), MK-2206 (AKT inhibitor), everolimus (mTOR inhibitor), or doxorubicin ${ }^{20,24}$. ASP ${ }^{\mathrm{cKO}}$ rhabdospheres displayed increased sensitivity to GDC-0941 but no difference in sensitivity to MK-2206, everolimus, or doxorubicin (Fig. 3c-f). Although $\mathrm{ASP}^{\mathrm{CKO}}$ tumors show increased phosphorylation of AKT, these results suggest the phenotypic differences observed in ASPcKO tumors are most likely mediated through PI3K or alternative PTEN functions.

$D b x 1$ is the most overexpressed gene in ASP ${ }^{c K O}$ tumors and has a functional role in human FN-RMS. Given PTEN nuclear localization in ASPWT tumors, we sought to determine if known nuclear functions were responsible for the ASPcKO phenotype. Neither DNA damage nor chromosome instability was increased in the ASPcKO tumors (Supplementary Fig. 5a-c). We next explored whether PTEN regulated gene expression and analyzed the gene expression changes in bulk ASPWT and ASPcKO tumors as well as SCM muscle. Principal component analysis revealed that gene expression in ASPWT and ASPcKO tumors clustered distinctly; however, they were more similar to each other than to mature SCM skeletal muscle (Fig. 3g and Supplementary Data 1). Comparing gene expression in ASP ${ }^{\mathrm{cKO}}$ to ASPWT tumors, 248 genes had increased and 286 genes had decreased expression ( $\geq$ twofold, $P$ value $<0.05$ ) (Fig. $3 \mathrm{~h}$ ). Several differentially expressed genes were validated by real-time PCR (Fig. 3i). Gene ontology analysis of the 286 genes with decreased expression in ASP ${ }^{\mathrm{cKO}}$ tumors compared to ASPWT tumors uncovered gene ontology (GO) terms associated with skeletal myogenic differentiation consistent with increased expression of embryonic MyHC and decreased expression of adult MyHC in ASPcKO tumors, and dysregulated immune responses (Figs. 2d-g and 3j); conversely, 248 genes with increased expression (Supplementary Data 1) revealed GO terms associated with increased proliferation and DNA replication consistent with increased Ki67 staining in ASPcKO tumors (Figs. 1e, f and 3k). Furthermore, we utilized CIBERSORT to analyze gene expression profiles from ASPWT and ASPCKO tumors to predict immune cell composition; ASPcKO tumors were predicted to have less naive B cells, M1 macrophages, resting mast cells, and eosinophils while M0 macrophages are enriched in the ASPcKO tumors (Supplementary Fig. 4d).

The subset of genes relatively overexpressed in ASPcKO tumors included neuronal transcription factors including Dlx5 and Pax7 as well as Dbx1 (Developing brain homeobox 1), the most overexpressed gene in the ASPcKO tumors (Fig. 4a). DBX1 is a homeobox transcription factor thought to function as a 
a

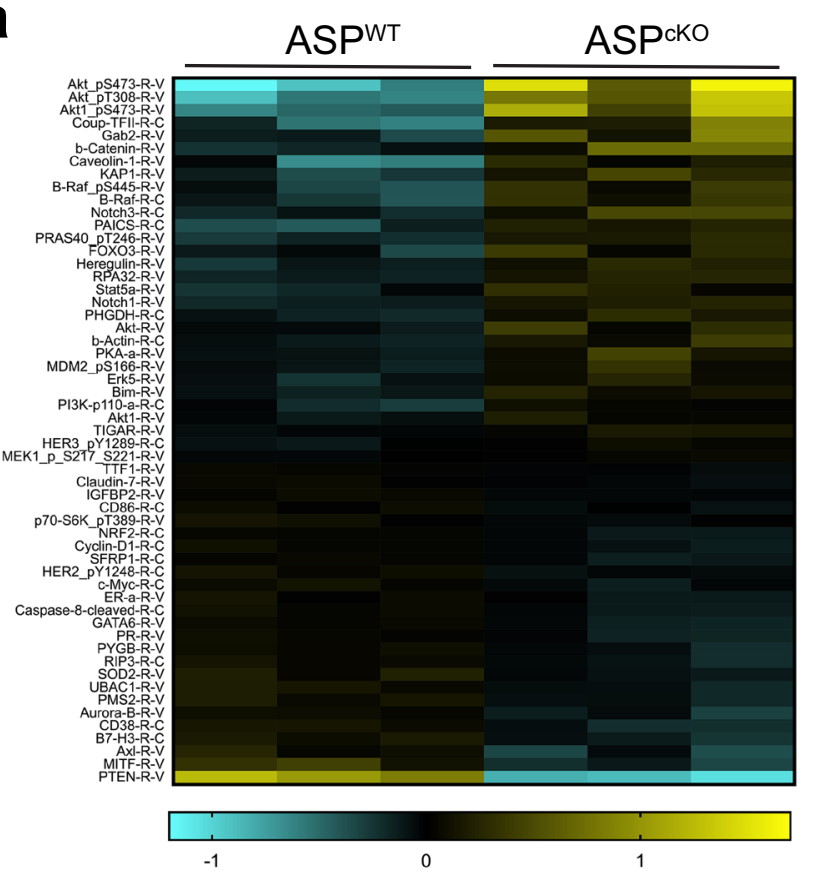

C

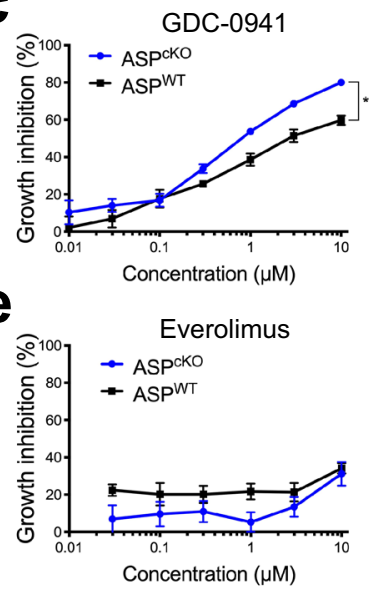

i

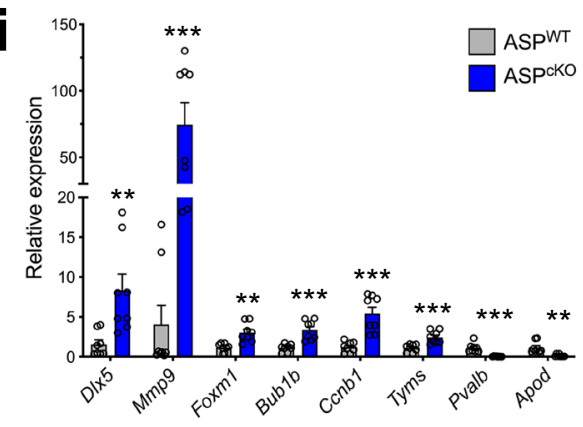

b
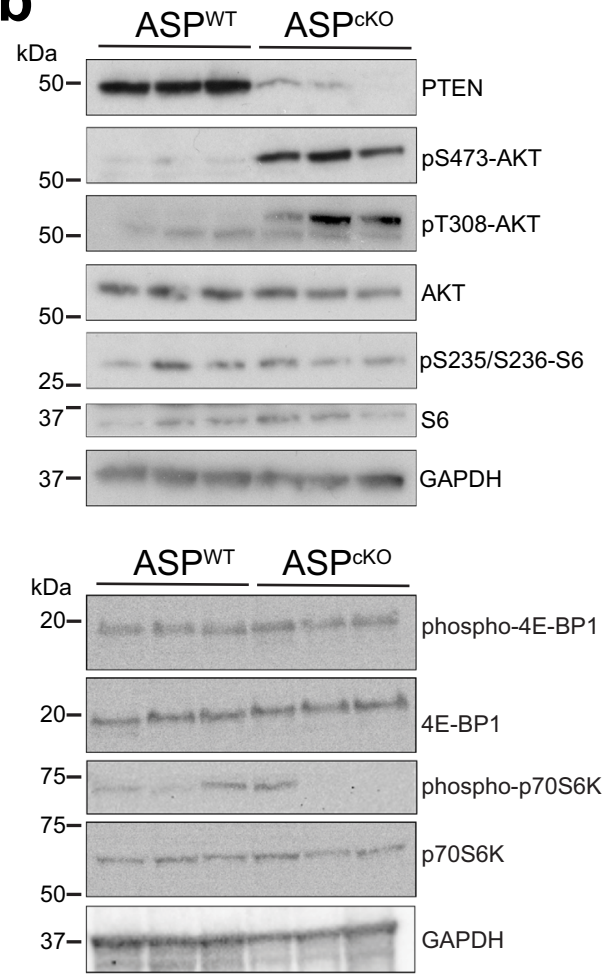

g
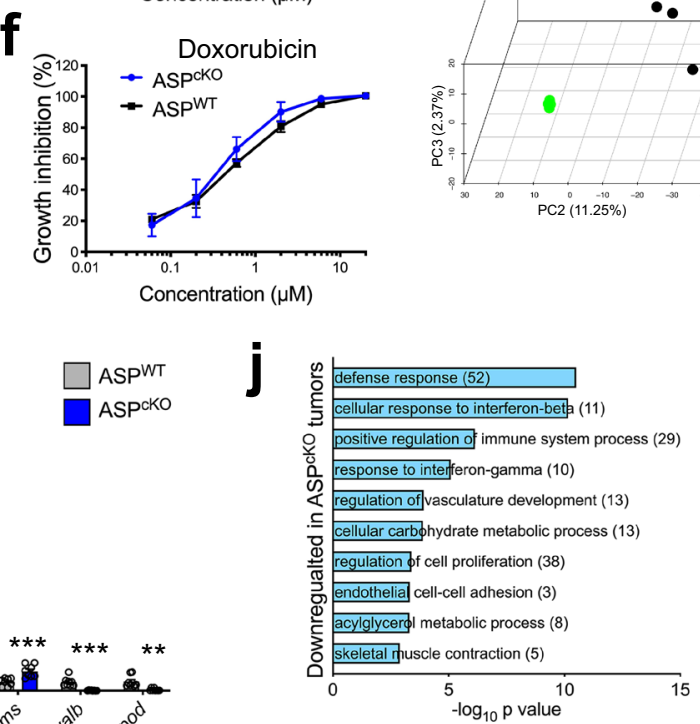

h

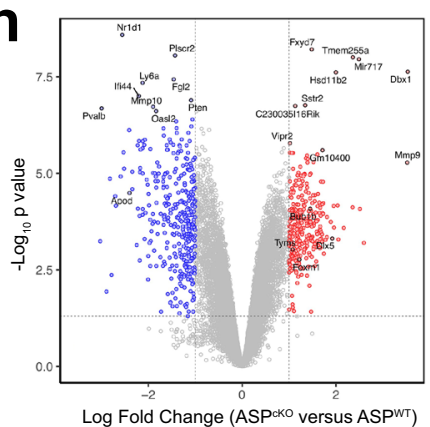

K

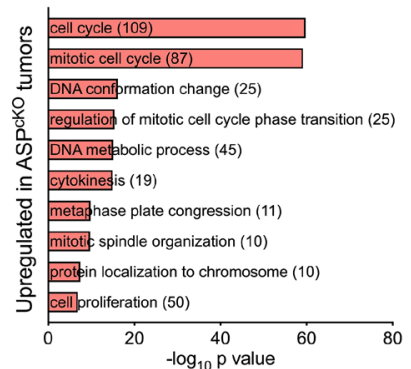

transcriptional repressor critical in neural patterning of innate processes $^{25-27}$. However, the role of DBX1 in cancer is unknown.

Given the lack of knowledge about DBX1's function in cancer, we first interrogated the Cancer Dependency $\mathrm{Map}^{28}$ and found that $D B X 1$ highly expressed in RMS cell lines with only rhabdoid tumors exhibiting higher expression (Fig. 4b). Next, we probed two public human RMS expression datasets both illustrating increased $D B X 1$ expression in FN-RMS (Fig. 4c and Supplementary Fig. 6a $)^{29,30}$. Furthermore, increased DBX1 expression was also seen in all FP-RMS and most FN-RMS patient-derived xenografts (PDX) from the Childhood Solid Tumor Network at St. Jude compared to primary human myoblasts (Fig. 4d) 31 Therefore, DBX1 expression is increased in both mouse and human FN-RMS; however, the functional significance of DBX1 in RMS biology remains unknown.

To study the role of DBX1 in human FN-RMS, we generated a cell line from FN-RMS PDX SJRHB015721_X1 with high DBX1 expression (Fig. 4d and Supplementary Fig. 6b, c). We transduced the cells with lentivirus expressing either two independent shRNAs targeting DBX1 or a scramble shRNA control. 
Fig. 3 PTEN loss activates AKT but not mTORC1 in ASPcKo tumors. a Heatmap of the 55 significantly $(P<0.05)$ different total and phosphoproteins by RPPA, normalized, log2 transformed and median-centered between ASPWT and ASPcKO tumors, $n=3$. $\mathbf{b}$ Immunoblots of PTEN, phosphorylated AKT Ser473 or AKT Thr308, AKT, phosphorylated S6Ser235/236, S6, phosphorylated-4E-BP1, 4E-BP1, phosphorylated p70S6K, p70S6K, and GAPDH, $n=3$. c-f CellTiterGlo growth assays of ASPWT or ASPcKO rhabdospheres treated with GDC-0941 (c) $(P=0.0020)$, MK-2206 (d), everolimus (e), or doxorubicin $(\mathbf{f}), n=3$, in triplicate. $\mathbf{g}$ Principal component analysis of transcriptional profiles from ASPWT (black) and ASPcKO (blue) tumors and Cre-negative SCM (green), $n=4$. $\mathbf{h}$ Volcano plot showing differentially expressed genes between ASPWT and ASPCKO tumors from ( $(\mathbf{g}$ ). Genes with increased (red) and decreased (blue) expression in ASPCKO tumors ( $\geq$ twofold, $P<0.05$ ) are highlighted. i Validation of gene expression in $(\mathbf{h})$ by real-time PCR $(n=3$ SCM, $n=4$ tumors, in duplicate), expression normalized to $18 \mathrm{~S}$ rRNA and expressed relative to ASPWT. ( $D / x 5 P=0.0062, M m p 9 P=0.0009$, Foxm1 $P=0.0011$, Bub1b $P=0.0002$, Ccnb1 $P=0.0002$, Tyms $P=0.0009$, Pvalb $P=0.0001$, Apod $P=0.0018)$. j, $\mathbf{k}$ Gene ontology $(G O)$ analyses of genes with increased (j) and decreased (k) expression in ASPCKO tumors versus ASPWT tumors. Top ten unique GO terms are shown. All $P$ values in pairwise comparisons were determined by Student's $t$ test (unpaired, two-tailed); ${ }^{\star \star} P<0.01,{ }^{\star \star \star} P<0.001,{ }^{\star \star \star \star} P<0.0001$. Data represented as mean \pm SEM. See also Supplementary Figs. 4 and 5 and Supplementary Data 1. Source data are provided as a Source Data file.

DBX1-targeting shRNAs decreased $D B X 1$ expression and cell viability compared to the scramble control, suggesting a role for DBX1 in FN-RMS (Fig. 4e, f). To further understand the functional role of DBX1 in RMS, we performed gene expression analysis on the SJRHB015721_X1 cell line transduced with the two shRNAs and scramble control. 197 genes had decreased expression and 156 genes had increased expression in both shRNAs compared to scramble control (absolute log fold change $>0.58,-\log _{10}$ adjusted $P<0.5$ ) (Fig. $4 \mathrm{~g}$ and Supplementary Fig. 6d and Supplementary Data 2). Gene ontology analysis of the 197 genes with decreased expression with DBX1 knockdown highlights processes related to cell proliferation (Fig. 4h and Supplementary Data 2). The GO terms of the 156 genes with increased expression upon $D B X 1$ knockdown were enriched for apoptotic processes as well as positive regulators of development and morphogenesis (Fig. 4i and Supplementary Data 2). These data suggest the DBX1 corepressor activity assists in maintaining the proliferative state and blocked differentiation in FN-RMS.

To determine the in vivo significance of DBX1, we dissociated two human FN-RMS PDX samples, SJRHB015721_X1 and SJRHB012405_X1, and transduced cells with lentiviral vectors expressing EGFP as a marker of transduction and shRNA to knockdown DBX1 expression. After transduction, cells were injected into the gastrocnemius of SCID/Beige mice and used to establish primary rhabdosphere cultures. DBX1 expression decreased with shRNA expression in rhabdospheres indicating efficient $D B X 1$ knockdown after viral transduction (Supplementary Fig. 6e, f). DBX1 knockdown in both SJRHB015721_X1 and SJRHB012405_X1 PDXs reduced tumor volume and tumor mass (Fig. 4j, k and Supplementary Fig. 6g, h, j, k). Gross and immunohistochemical analysis indicated that the DBX1 knockdown tumors were indistinguishable from the scramble controls except that the $\operatorname{sh} D B X 1$ tumors that formed lost EGFP expression (Fig. 41 and Supplementary Fig. 6i, 1). This suggests that tumors developing from shDBX1-transduced cells had escaped lentiviral transduction and thus escaped DBX1 knockdown highlighting the importance of DBX1 in FN-RMS.

PAX7 controls Dbx1 expression. Given $D b x 1$ is the most overexpressed gene in ASPCKO tumors and DBX1 knockdown decreased tumor growth in human FN-RMS cells, we sought to determine how Pten loss regulates Dbx1 expression. We first examined a $1 \mathrm{~kb}$ promoter region immediately 5' of Dbxl's initiating ATG to identify known transcription factor binding motifs and compared this to genes with >twofold expression in ASPcKO tumors and found only one overlapping gene - Pax7 (Fig. 5a). PAX7 is a transcription factor critical in both adult muscle stem cell (satellite cells) maintenance and preserving a dedifferentiated state in FN-RMS ${ }^{32,33}$. Interestingly, PAX7 along with MYOD1 are the only genes that are both members of FNRMS core-regulatory circuits and are FN-RMS dependencies thus coupling tumor cell identity and survival (Fig. 5b) ${ }^{34,35}$. We confirmed increased Pax7 expression in ASPCKO tumors compared to ASPWT tumors by real-time PCR, immunoblotting, and immunofluorescence (Fig. $5 \mathrm{c}-\mathrm{f}$ ). To confirm the link between PTEN and Pax7 transcription, we infected Pten flox mouse embryonic fibroblasts (MEFs) with adenovirus encoding either Cre recombinase or GFP. Adeno-Cre-infected Ptenflox MEFs exhibited decreased Pten expression and increased Pax7 (Fig. 5g). Next, to determine if PAX7 transcriptionally regulates $D b \times 1$, we cloned 900 bp of the Dbx1 promoter containing a putative PAX7binding site upstream of luciferase. PAX7 expression led to a dose-dependent increase in Dbx1-promoter-driven luciferase activity (Fig. 5h, i). Next, we transduced wild-type MEFs with lentivirus overexpressing PAX7 and showed concomitant increased $D b x 1$ expression (Fig. 5j). Conversely, shRNA knockdown of Pax7 in ASPCKO rhabdospheres resulted in decreased Dbx1 expression (Fig. 5k). Furthermore, decreased PAX7 expression in human rhabdospheres led to decreased $D B X 1$ expression (Fig. 5l).

The murine and human DBX1 proximal promoters are highly conserved (Fig. $5 \mathrm{~m}$ ). Of the two putative PAX7-binding sites within the human $D B X 1$ promoter, only the proximal (site 1) is conserved in the mouse. We designed primers that flank each of these human sites and performed chromatin immunoprecipitation (ChIP)-qPCR to analyze whether PAX7 is bound to the $D B X 1$ promoter. PAX7 binding was enriched eightfold over control IgG at the proximal $D B X 1$ promoter PAX7-binding motif (which also corresponds to the mouse $D b x 1$ promoter) (Fig. $5 \mathrm{n}$ ). These data indicate PTEN loss increases PAX7 expression and DBX1 is a downstream target of PAX7 in FN-RMS.

Human FN-RMS is dependent on PAX7. Given that PAX7 is putatively both a transcription factor in the RMS core-regulatory circuit and a dependency in RMS, we sought to further explore the role of PAX7 in RMS. The Broad Institute's Cancer Dependency Map (DepMap) illustrates that $P A X 7$ is highly expressed in soft-tissue sarcoma cell lines (Fig. 6a) ${ }^{28}$. Specifically, PAX7 expression is increased in human FN-RMS tumors compared to skeletal muscle in a public human RMS expression dataset (Fig. 6b) ${ }^{30}$, as well as in human FN-RMS cell lines and PDXs compared to myoblasts (Supplementary Fig. 7a, b). CRISPR/ Cas9-dependency screening in DepMap revealed a PAX7 dependency in FN-RMS cell lines (Fig. 6c). Thus, we wanted to confirm if human FN-RMS cell lines and PDXs are dependent on PAX7 expression. Using lentiviruses expressing two independent shRNA targeting $P A X 7$ or a scramble control, we performed $P A X 7$ knockdown in human FN-RMS cell lines (SMS-CTR, RD, Rh18) and cell lines derived from two PDXs - SJRHB015721_X1 and SJRHB011_Y (Fig. 6d and Supplementary Fig. 7c, d). $P \bar{A} X 7$ knockdown in FN-RMS cells decreased both cell viability and cell 

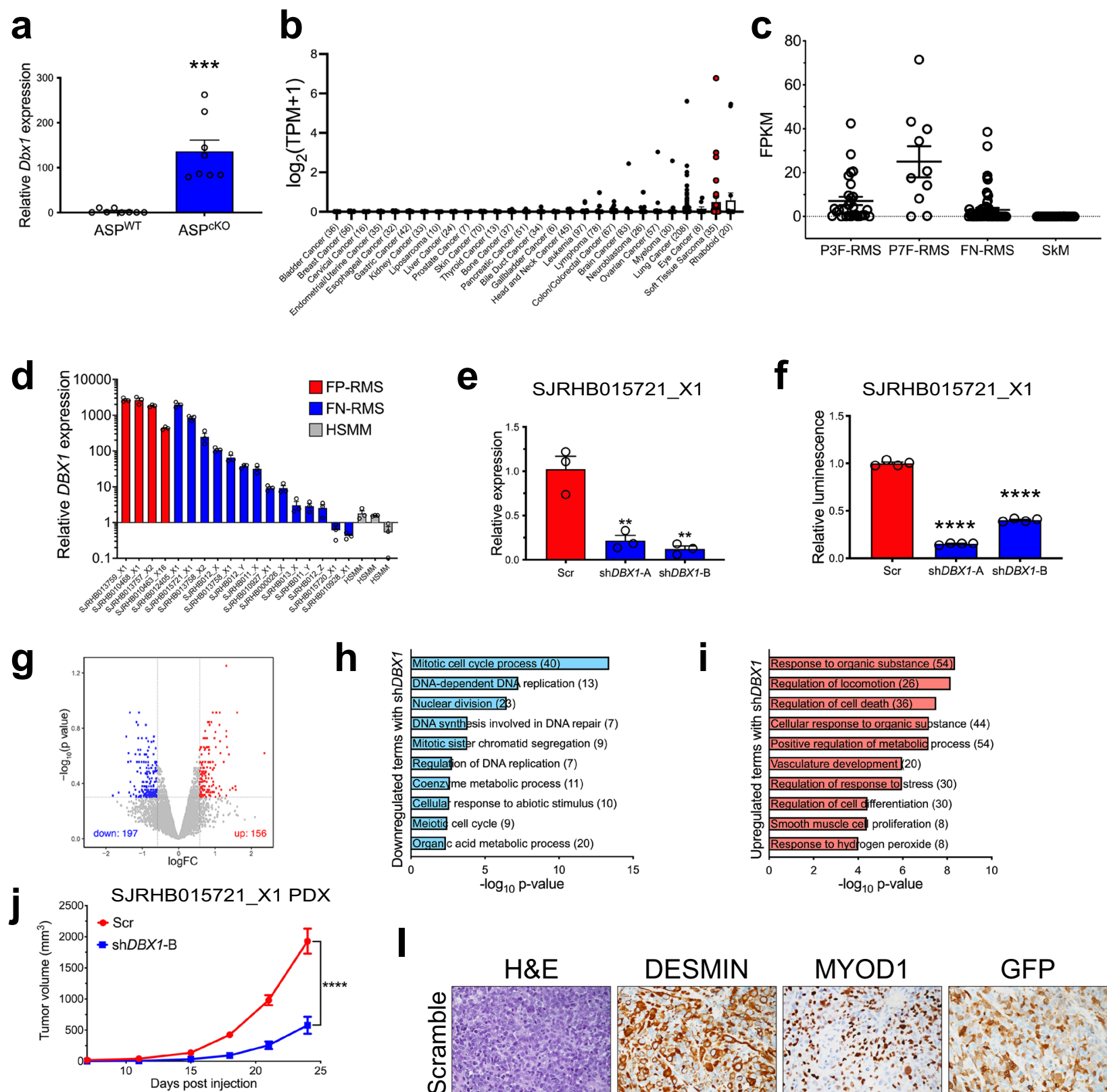

$\mathbf{k}$
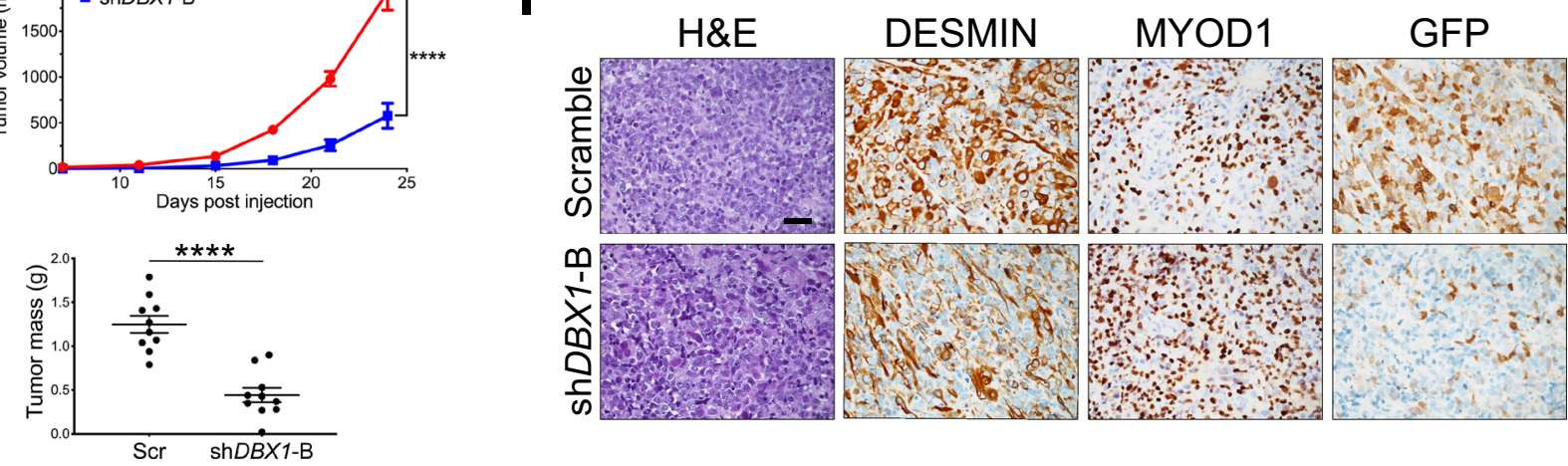

growth in vitro suggesting a role in FN-RMS (Fig. 6e, $\mathrm{f}$ and Supplementary Fig. 7e-h).

Since $P A X 7$ knockdown reduced FN-RMS cell proliferation in vitro, we aimed to determine whether $\operatorname{sh} P A X 7$ knockdown affected tumorigenicity in vivo. SMS-CTR cells transduced with shPAX7 or scramble control were injected into the gastrocnemius of SCID/Beige mice. PAX7 knockdown significantly reduced tumor volume and mass in SMS-CTR cell xenografted mice (Fig. 6g, h, i). To establish this was not a consequence or artifact of using an established cell line, we extended our analysis to FNRMS PDXs. SJRHB015721_X1 and SJRHB012405_X1 PDX cells were dispersed into single cells and transduced with lentivirus expressing $\operatorname{sh} P A X 7$ or scramble control. After a brief incubation at $37^{\circ} \mathrm{C}$, the cells were either placed in spheroid culture or injected into the gastrocnemius muscle of SCID/Beige mice. PAX7 knockdown was confirmed in spheroid culture cells (Supplementary Fig. 7i). In both SJRHB015721_X1 and SJRHB012405_X1 PDXs, PAX7 knockdown with shPAX7 resulted in decreased tumor volume (Fig. 6j and Supplementary Fig. 7j); however, tumor mass only decreased in SJRHB015721_X1 (Fig. 6k and Supplementary Fig. 7k). Tumors from SJRHB012405_X1 retained PAX7 expression at the endpoint suggesting a negative selection for cells with PAX7 knockdown (Fig. 61 and Supplementary Fig. 7l). Together, our in vitro and in vivo data confirm $P A X 7$ is a dependency in FNRMS cells. 
Fig. 4 Dbx1 is the most overexpressed gene in ASPcKO tumors and has a functional role in human FN-RMS. a Gene expression by real-time PCR of $D b x 1$ $(P=0.0001)$ in ASPWT tumors $(n=4)$ ASPcKO tumors $(n=4)$, in duplicate. b DBX1 mRNA expression $\left(\log _{2}(\right.$ TPM +1$\left.)\right)$ in cell lines from the Broad Institute Cancer Dependency Map (DepMap), 20 Q3 release. Soft-tissue sarcoma cell lines are in red. The number of cell lines is indicated along the $x$ axis label for each respective tumor type. c DBX1 expression by RNA sequencing (FPKM) in human FN-RMS $(n=64)$, FP-RMS (PAX3-FOXO1 (P3F, $n=29)$ and PAX7-FOXO1 (P7F, $n=10)$ ) tumors, and human skeletal muscle samples and human myoblast tissue cultures $(n=26)(\mathrm{SkM})$ from the NCl30. d Gene expression by real-time PCR of DBX1 in RMS PDXs and human skeletal muscle myoblasts (HSMMs), in triplicate. e Gene expression by real-time PCR of DBX1 in SJRHB015721_X1 cell line following lentiviral transduction (72 h) with DBX1-targeting or control shRNA (Scr), in triplicate. (Scr-shDBX1-A

$P=0.0068$, Scr-shDBX1-B $P=0.0039)$. f Cell viability by Cell Titer Glo assay of SJRHB015721_X1-derived adherent cell cultures transduced with DBX1targeting or control shRNAs after 5 days; in quadruplicate, compared to scramble. (Scr-shDBX1-A $P<0.0001, S c r-s h D B X 1-B P<0.0001$ ). $\mathbf{g}$ Volcano plot depicting differentially expressed genes between two $D B X 1$-targeted and control shRNAs (absolute log fold change $>0.58,-\log _{10}$ (adjusted $P<0.5$ ) in SJRHB015721_X1 cells. h, $\mathbf{i}$ GO analyses of genes with decreased (h) and increased (i) expression following shRNA-mediated DBX1 depletion in the SJRHB015721_X1 cell line. Top ten unique GO terms are shown, DAVID analysis. j, k Tumor volume of SJRHB015721_X1 orthotopic patient-derived xenograft (PDX) tumor cells transduced with DBX1-targeting or control shRNAs $(\mathbf{j}, P<0.0001)$ and tumor mass $(\mathbf{k}, P<0.0001), n=10$. I Representative H\&E staining and IHC for DESMIN, MYOD1, and GFP from tumors in $(\mathbf{j})$. Scale bar $=25 \mu \mathrm{m}$. All $P$ values in pairwise comparisons were determined by Student's $t$ test (unpaired, two-tailed); ${ }^{\star \star} P<0.01,{ }^{\star \star \star \star} P<0.0001$. Data represented as mean \pm SEM. Real-time PCR normalized to $18 \mathrm{~S} r \mathrm{RNA}$ and expressed relative to $\operatorname{ASPWT}$ (a), the average of the HSMM cell lines (d), or scramble (e). See Supplementary Fig. 6 and Supplementary Data 1 and 2. Source data are provided as a Source Data file.

PAX7 dictates rhabdomyosarcoma tumor cell identity in ASPcKO tumors. To elucidate the mechanistic role of PAX7 in FN-RMS, we returned to our ASP ${ }^{\text {CKO }}$ mouse model. We bred Paxflox alleles into the ASP ${ }^{\mathrm{CKO}}$ model to generate compound conditional knockout mice, aP2-Cre;Smo ${ }^{M 2 /+}$;Pten flox/flox Pax7flox/flox (ASPcKOP7cKO) and associated littermate control mice (Fig. 7a). Deleting Pax7 in ASPCKO mice rescued the decreased tumor-free survival and increased tumor mass associated with Pten loss in the AS mice (Fig. $7 \mathrm{~b}, \mathrm{c}$ ). ASP ${ }^{\mathrm{cKO}} \mathrm{P} 7^{\mathrm{cKO}}$ tumors were noncircumscribed and highly infiltrative with the tumor cells perpendicular to and through the skeletal muscle fibers (Fig. 7d). Histologically, the ASP ${ }^{\mathrm{cKO}}{ }^{\mathrm{P}} \mathrm{CKO}^{\mathrm{C}}$ tumor cells stained sporadically for DESMIN but were no longer positive for RMS diagnostic markers MYOD1 and MYOGENIN; this was specific for $\mathrm{ASP}^{\mathrm{cKO}} \mathrm{P}^{\mathrm{cKO}}$ tumors as ASPcHetP7cKO and ASPcKOP7 ${ }^{\mathrm{cHet}}$ tumors were still myogenic, small round blue tumors consistent with RMS (Fig. 7d). The ASPcKOP7cKO tumors were also significantly less proliferative than either ASPWT and ASPcKO tumors (Fig. 7e). However, these phenotypes cannot be attributed to differences in PI3K-pathway activation observed in ASPcKO and ASPWT tumors as ASPCKOP7cKO tumors had similar AKT and S6 phosphorylation as ASPcKO tumors (Fig. 7f). This highlights a unique link between PTEN and PAX7 independent of PI3K-pathway signaling that illustrates PAX7 is a synthetically essential gene in PTEN-deficient FN-RMS.

To determine the tumor type produced following concomitant Pten and Pax7 depletion, we first performed ultrastructural analysis and that revealed ASP ${ }^{\mathrm{CKO}} \mathrm{P} 7 \mathrm{cKO}$ tumor features were no longer consistent with skeletal muscle but with leiomyosarcoma (LMS), including elongated cellular shape, grooved nuclei, and prominent rough endoplasmic reticulum (Fig. 8a). Intriguingly, ASPcKOP7cKO tumors but not ASPWT or ASPcKO tumors expressed the LMS markers smooth-muscle actin (SMA) and CALDESMON (Fig. 8b). Given that ASP ${ }^{\mathrm{cKO}} \mathrm{P} 7^{\mathrm{cKO}}$ tumors had a lower proliferative index (Fig. 7e) and did not engraft into SCID/ Beige immunocompromised mice ( $0 / 6$ tumors), the tumors that arise may lie along a spectrum of low-grade sarcomas with smooth-muscle immunophenotypic features. These results illustrate that the Pten loss phenotype is dependent on PAX7 and indicates that PAX7 controls FN-RMS cell fate.

To explore the mechanisms altering ASPcKOP7cKO tumor identity, we compared the gene expression in ASPcKO and ASPcKOP7 ${ }^{\text {cKO }}$ tumors. We bred the Rosa26 tdTomato reporter mouse into our ASPcKOP7cKO model to generate ASTPcKOP7cKO compound mutant mice allowing us to use flow cytometry to purify tumor cells by sorting tdTomato-positive and CD31
(PECAM)-negative (to remove endothelial cells) cells (Supplementary Fig. 8a) ${ }^{20}$. RNAseq from sorted $\mathrm{Tom}^{+} \mathrm{CD} 31^{-}$tumor populations revealed robust differential gene expression with 973 genes enriched in ASTPCKO and 1002 genes enriched in ASTPcKOP7cKO tumor cells ( $\geq$ twofold, $P<0.05$ ) (Fig. $8 \mathrm{c}, \mathrm{d}$ and Supplementary Data 3 ). Functional annotation clustering and gene set enrichment analyses further highlighted differences between $\mathrm{ASTP}^{\mathrm{cKO}}$ and $\mathrm{ASTP}^{\mathrm{cKO}} \mathrm{P}^{\mathrm{cKO}}$ tumors with myogenic and neurogenic transcriptional programs enriched in ASTPcKO and relatively underexpressed in ASTPcKOP7 $\mathrm{CKO}$ (Fig. 8e, f, h, i and Supplementary Data 3). This dual regulation of skeletal myogenic and neurogenic genes is important to note as PAX7 is a transcription factor that regulates both developmental programs ${ }^{36,37}$. Curiously, all five distal-less homeobox genes were also relatively underexpressed in $\mathrm{ASTP}^{\mathrm{cKO}} \mathrm{P} 7 \mathrm{cKO}$, indicating PAX7 as a master regulator of their expression (Fig. 8e).

In contrast to the increase in skeletal myogenic and neurogenic transcriptional programs enriched in the ASTPcKO tumors, there were relatively few transcriptional programs increased in ASTPcKOP7cKO tumors (Fig. 8e, g, h, j and Supplementary Data 3). The major pathways enriched in ASTPcKOP7cKO tumors included calcium signaling, cyclic nucleotide pathways, and most intriguingly, vascular smooth muscle including the smooth muscle master-regulator transcription factor Myocardin $(\text { Myocd })^{38}$. Furthermore, ASTP ${ }^{c K O} 7^{c K O}$ tumors were enriched for genes associated with LMS, including smooth-muscle structural proteins Acta2 and Myh11. Most of the LMS genes (Lmod1, Casq2, Actg2, Mylk, and Slmap) are markers of subtype I of human LMS which is mostly found in extrauterine sites ${ }^{39}$. Unlike the expression differences in Lmod1, Myh11, and Acta2 observed between ASP ${ }^{\mathrm{cKO}} 7^{\mathrm{cKO}}$ and ASPcKO tumors (Supplementary Fig. 8b), we did not find any comparable increases in LMOD1, MYH11, and ACTA2 following shRNAmediated knockdown of PAX7 expression in three human FN-RMS cell lines/PDXs-SMS-CTR, SJRHB015721_X1, and SJRHB012405_X1-highlighting that this PAX7-controlled fate decision occurs early in tumorigenesis (Supplementary Fig. 8c-e). Furthermore, tumors that form in xenografts of SMS-CTR cell line and the SJRHB015721_X1 and SJRHB12405_X1 PDXs following PAX7 knockdown have not shifted fate to LMS by IHC for SMA and CALDESMON (Supplementary Fig. 8f). These results indicate that deletion of $\operatorname{Pax} 7$ in the ASPcKO murine FNRMS model yields tumors whose histology, ultrastructure, and gene expression recapitulates low-grade sarcoma with smoothmuscle differentiation and not a skeletal myogenic tumor-like FN-RMS. Therefore, simultaneous Pten and Pax7 loss in murine 

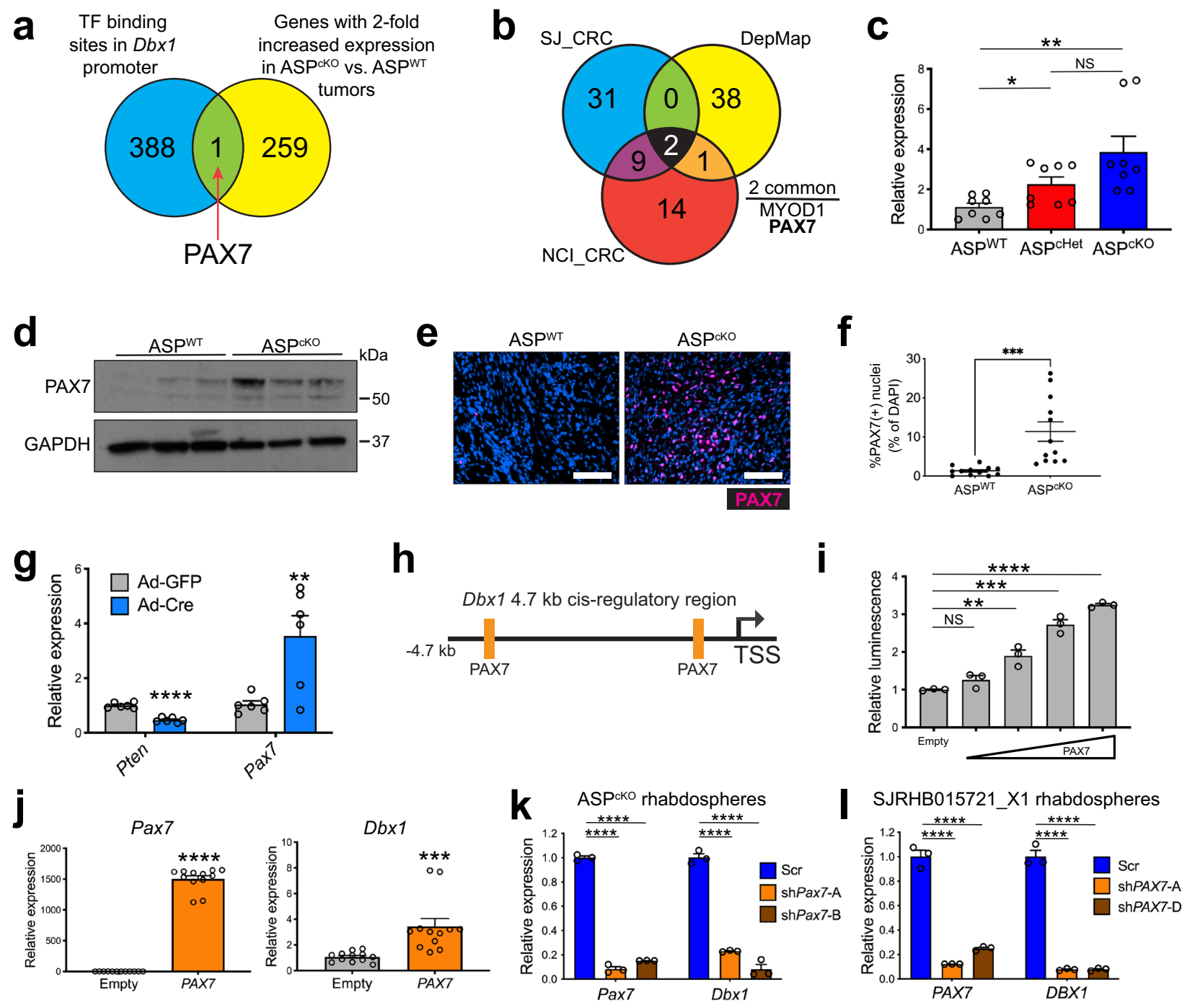

m
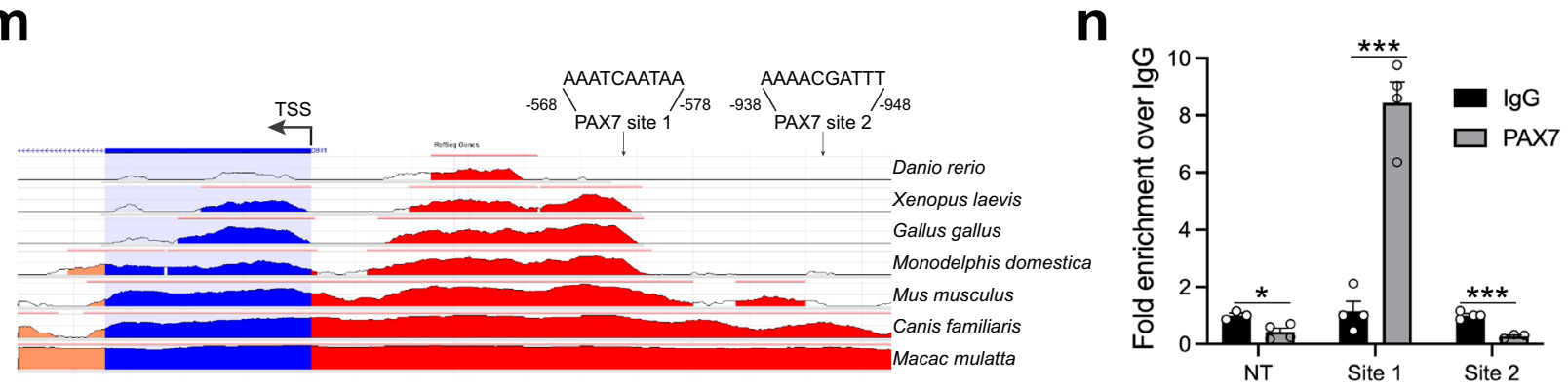

FN-RMS not only rescued the effects of Pten loss in these tumors but identified a synthetic essential relationship between Pten and Pax7 controlling tumor cell fate.

\section{Discussion}

The cell of origin and genetic perturbations cooperate in tumor cell transformation and fate determination highlighting cellular and developmental pliancy in tumorigenesis. Genetically engineered mouse models offer a method to dissect these relationships by utilizing aP2-Cre to activate oncogene and/or delete tumor suppressor expression. Genetic fate mapping reveals aP2Cre expression in white and brown adipocytes, dorsal root ganglia, macrophages, and endothelial cells ${ }^{20,22,40}$. Compound mutant mice using aP2-Cre to drive recombination resulting in biallelic Dicer1 deletion or the combination of oncogenic
Kras $^{\mathrm{G} 12 \mathrm{D}}$ activation and Cdkn2a deletion result in angiosarcoma from the transformation of endothelial cells ${ }^{20,41}$. However, activation of constitutively active, oncogenic $\mathrm{SMO}^{\mathrm{M} 2}$, and hedgehog pathway signaling by aP2-Cre results in cell reprogramming and transformation of endothelial progenitor cells driving FN-RMS tumorigenesis ${ }^{20}$. We now find Pten loss cooperates with $\mathrm{SMO}^{\mathrm{M} 2}$ in aP2-Cre expressing cells to drive a less differentiated tumor that better recapitulates the most common human embryonal RMS histology. This was specific to Pten loss as $C d k n 2 a, R b 1$, or Trp53 loss did not phenocopy the results. The effects of Pten deletion were dependent on PAX7 as Pax7 deletion rescued the phenotypes of Pten loss and altered tumor cell fate resulting in a spectrum of low-grade sarcomas with immunophenotypic smooth-muscle differentiation, including LMS. Interconnections between RMS and LMS have been described 
Fig. 5 PAX7 controls Dbx1 expression. a Venn diagram depicting Pax7 as the sole overlapping gene between transcripts enriched in ASPcKO tumors and transcription factors (TFs) with putative binding sites (from JASPAR 2016) ${ }^{72}$ within the Dbx1 promoter (1 kb upstream of TSS). b Venn diagram depicting the overlap between core transcriptional circuitry genes and FN-RMS DepMap (Cancer Dependency Map) gene dependencies ${ }^{28,31,35}$. c Gene expression by real-time PCR of Pax7 in ASPWT and ASPcKO tumors. Expression normalized to Actb and expressed relative to ASPWT, $n=4$, in duplicate. (ASPWTASPcHet $P=0.0123$, ASPWT-ASPCKO $P=0.0046)$. $\mathbf{d}$ Immunoblot of PAX7 and GAPDH in ASPWT and ASPcKO tumors lysates, $n=3$. e, $\mathbf{f}$ Representative IF depicting increased PAX7 (red) staining in ASPCKO tumors versus ASPWT tumors (DAPI nuclear stain, blue) (e) quantitated in (f), $n=3,12$ fields of view per genotype, $P=0.0006$. Scale bar $=25 \mu \mathrm{m}$. $\mathbf{g}$ Gene expression by real-time PCR of Pax 7 and Pten following $72 \mathrm{~h}$ post-adenoviral-Cre infection of $P$ ten ${ }^{\text {flox } /}$ flox mouse embryonic fibroblasts (MEFs). Expression normalized to $18 \mathrm{~S}$ rRNA and expressed relative to Ad-GFP-infected cells, $n=3$ independent MEF cultures, in duplicate. (Pten $P<0.0001, P a x 7 P=0.0080$ ). h Cartoon illustrating putative PAX7-binding sites in the $4.7 \mathrm{~kb} D b \times 1$ cis-regulatory region upstream of transcription start site (TSS). i Representative Dbx1-promoter-luciferase assay depicting a PAX7 dose-dependent increase in luciferase activity. $n=3$ independent assays, in technical triplicate. (empty $250 \mathrm{ng} P=0.0043$, empty $500 \mathrm{ng} P=0.0002$, empty $750 \mathrm{ng} P<0.0001$ ). j Gene expression by real-time PCR of $\operatorname{Dbx} 1(P=0.0009)$ and $\operatorname{Pax} 7(P<0.0001)$ following stable transduction of wild-type MEFs with Pax7. Expression normalized to 18S rRNA and relative to empty control, $n=6$ independent MEF cultures, in duplicate. k Gene expression by real-time PCR of Dbx1 and Pax7 in primary ASPcKO rhabdospheres transduced with Pax7-targeting or scramble shRNAs, 5 days post transduction, in triplicate. Normalized to Actb expression and expressed relative to scramble. All $P$ values $<0.0001$. I Gene expression by real-time PCR of DBX1 and PAX7 in SJRHB015721_X1 patient-derived rhabdospheres transduced with $P A X 7$ targeting or control harvested 8 days post transduction, in triplicate. Normalized to $18 \mathrm{~S}$ expression and expressed relative to scramble. All $P$ values $<0.0001$. $\mathbf{m}$ Evolutionary conservation of the human DBX1-promoter region (1552 bp depicted from within the first intronic region through first 1000 bp upstream of the DBX1 transcription start site) as obtained from the Evolutionary Conserved Regions database

(ecrbrowser.dcode.org) ${ }^{71}$. Pink bars on top of each species track show conservation to the human sequence. Salmon coloring indicates conserved intronic regions, blue indicates conserved protein-coding sequences, and red indicates conserved intergenic regions. Also depicted are the two putative PAX7binding sites (with binding sequence) from the JASPAR ${ }^{73}$ database within the DBX1-promoter sequence. All species compared to the human DBX1 DNA sequence. $\mathbf{n}$ ChIP-PCR of PAX7 binding to the proximal site 1 and not distal site 2 within the DBX1 promoter in SJRHB015721_X1 cells. NT = non-PAX7binding amplicon within the $D B X 1$ promoter; two biologically independent samples performed in duplicate. Normalized to IgG binding at respective sites. (NT $P=0.0074$, site $1 P=0.0001$, site $2 P=0.0003$ ). All $P$ values in pairwise comparisons were determined by Student's $t$ test (unpaired, two-tailed); ${ }^{\star} P<0.05,{ }^{\star \star} P<0.01,{ }^{\star \star \star} P<0.001,{ }^{\star \star \star \star} P<0.0001$. Data represented as mean \pm SEM. Source data are provided as a Source Data file.

with anecdotal clinical reports of LMS co-occurring with RMS or with rhabdomyosarcomatous-like lesions ${ }^{42,43}$. This suggests there may be some shared developmental trajectories and highlights the fluidity of cellular and developmental pliancy in tumorigenesis.

$D b x 1$ was the most relatively overexpressed gene in ASPcKO tumors and has not been reported to have a role in cancer. DBX1 is an evolutionarily conserved homeobox transcription factor expressed embryonically in central nervous tissue functioning as a transcriptional corepressor to establish specific neuron fate and neural patterning ${ }^{25-27}$. We found that both human FN-RMS and FP-RMS had subsets of tumor with high DBX1 expression. Interestingly, DBX1 transcription was controlled by the skeletal muscle satellite cell marker PAX7 in both murine and human FNRMS models. Since FP-RMS tumors are driven by PAX3-FOXO1 or PAX7-FOXO1 fusion oncoproteins containing either PAX3 or PAX7 DNA binding domains, we postulate that $D B X 1$ expression is driven transcriptionally by the fusion oncoproteins much how PAX7 itself targets DBX1 expression in FN-RMS. This may suggest some shared etiology between two major RMS subtypes.

Importantly, PAX7 not only functions in skeletal muscle development and maintenance of satellite cell quiescence but also other cell types, including pituitary gland melanotropes, neural tube, and actively migrating neural crest cells ${ }^{36,37,44,45}$. ASPcKO tumors engage a transcriptional program composed of several neural-associated genes, many of which are under the control of PAX7 as their expression is also lost in ASPcKOP7cKO tumors. Furthermore, neural stem cells have the ability to convert to a myogenic lineage and the myogenic determination factor MYOD1 can bind to neural-specific genes ${ }^{46,47}$. It has also been shown that Pten loss drives $\operatorname{Pax} 7$ mRNA increases in human neural stem cells further highlighting how transcriptional regulation by PTEN may be a critical mediator of its tumor suppressor functions ${ }^{48}$. This may provide therapeutic vulnerability as it appears that FN-RMS cells are dependent on these lineage factors 35,49

Engaging the PAX7 transcriptional program, such as with DBX1, in these FN-RMS tumors by Pten loss highlights the necessity in understanding the complete physiological consequences of PTEN loss in tumors. This includes potential changes in the immune microenvironment as our RPPA data suggest a more immunosuppressive environment, much like other tumors with PTEN loss ${ }^{50-52}$. Furthermore, increasing evidence indicates that PTEN mediates its tumor-suppressive effects via not only PI3K-pathway-dependent mechanisms but also through a myriad of PI3K-pathway-independent mechanisms ${ }^{11,53}$. PTEN-deficient cancers are known to have synthetic essential relationships with CHD1 and PDHK1 $1^{17,18}$. Concomitant Pax7 and Pten loss completely rescued the deleterious effects of the latter in our FN-RMS model suggesting Pten loss contributes to rhabdomyosarcomagenesis in a PI3Kpathway-independent mechanism via a synthetic essential interaction. It remains unclear how pervasive these synthetic essential relationships between tumor suppressor and lineage factors are in FN-RMS or other tumors. PAX7 is only one component of the FN-RMS core-regulatory circuits (CRCs) ${ }^{34,35}$. Do other components of the FN-RMS CRC phenocopy this result? Furthermore, other CRCs in additional tumor types are becoming clearer; do similar mechanisms underlying genetic alteration context, cell type, and transcriptional states give rise to lineage determinants and dependencies? These are open questions that need to be empirically explored.

The combination of near-universal PTEN perturbation in human FN-RMS and PI3K-pathway-independent mechanisms in maintaining tumor identity in murine FN-RMS indicates PTEN's role in activating RMS-specific transcriptional outputs. This work intertwines the interplay between context-dependent tumor suppressor loss and transcriptional activation can engage a synthetic essential pairing; here, the combination of PTEN loss and PAX7 activation not only maintains tumor growth and survival but maintains tumor identity. Here, we show that the ASP ${ }^{\mathrm{CKO} P 7^{\mathrm{CKO}}}$ tumors are no longer FN-RMS. The concomitant loss of PTEN and PAX7 gave rise to smoothmuscle-like tumors that lie along a spectrum of smooth myogenic differentiation from benign smooth-muscle-like tumors to those that appear like leiomyosarcoma. It is possible that the dual 

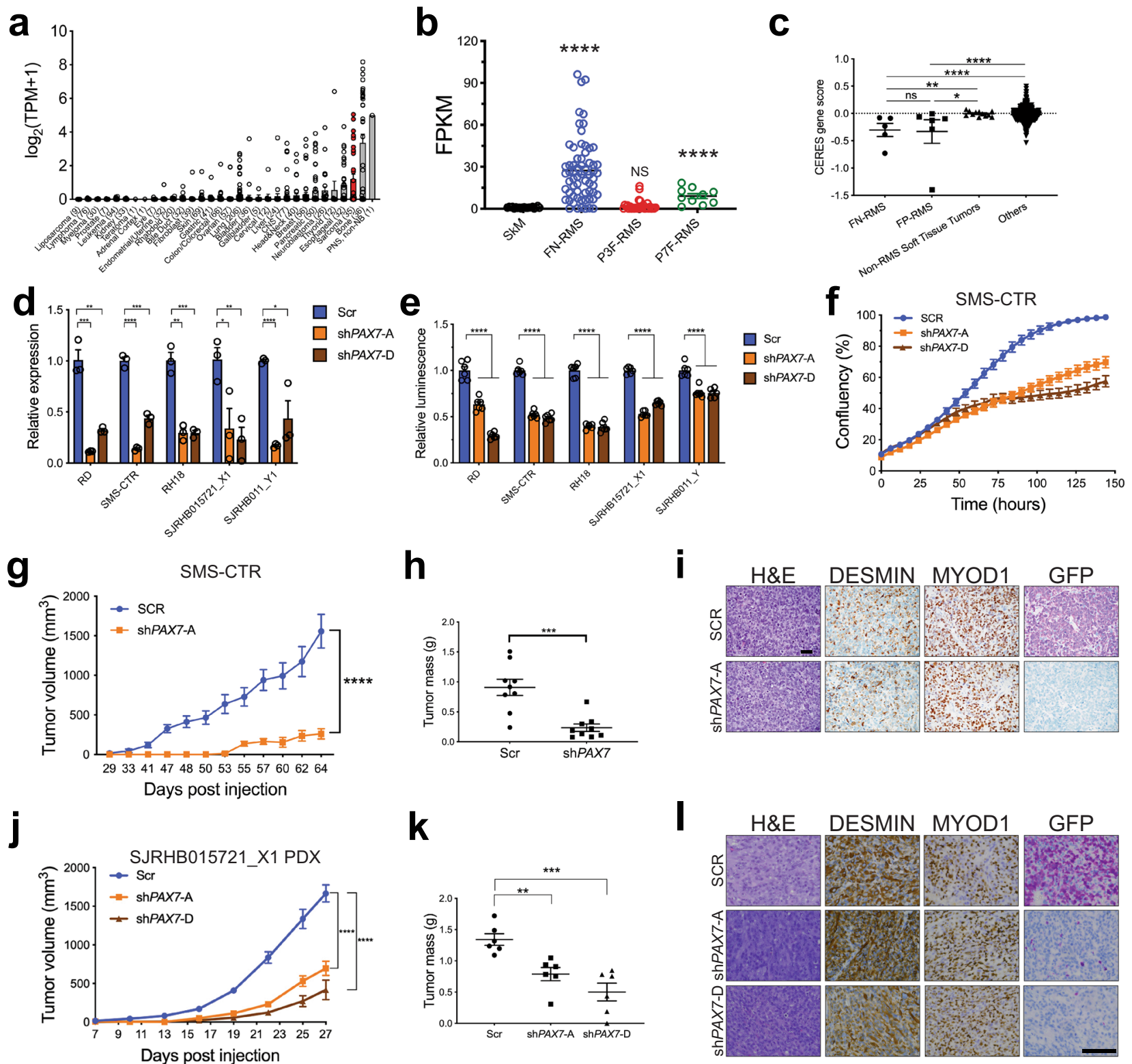

Fig. 6 Human FN-RMS is dependent on PAX7. a PAX7 expression by RNA sequencing $\left(\log _{2}(T P M+1)\right.$ ) from the DepMap (20Q3 release). Red bar indicates sarcoma cell lines. The number of cell lines is indicated along the $x$ axis label for each respective tumor type. b RNA sequencing (FPKM) of PAX7 in human FN-RMS $(n=64)$, FP-RMS (P3F-RMS $n=29$, P7F- RMS $n=10)$ tumors, and human skeletal muscle $(n=20)(\mathrm{SkM})$ from the NCl dataset ${ }^{30}$. (SkM-FN-RMS $P<0.0001$, SkM-P7F-RMS $P<0.0001$ ). c CERES gene dependency score for PAX7 in FN-RMS cells $(n=5)$ compared to all other cell lines in the DepMap, $20 \mathrm{Q} 3$ release. (FN-RMS-non-RMS soft-tissue tumors $P=0.0015$, FP-RMS-non-RMS soft-tissue tumors $P=0.0450, \mathrm{FN}$-RMS-others and FP-RMS-others $P<0.0001)$. d Gene expression by real-time PCR of PAX7 in SMS-CTR, RD, Rh18, SJRHB015721_X1, and SJRHB011_Y1-cultured cells following transduction with $P A X 7$ targeting or scramble shRNA, in triplicate, 5 days post transduction. Normalized to ACTB expression and expressed relative to scramble. (RD Scr-shPAX7-A $P=0.008$, RD Scr-shPAX7-D $P=0.0023$ ) (SMS-CTR Scr-shPAX7-A $P<0.0001$ Scr-shPAX7-D $P=0.0003$ ) (Rh18 Scr-shPAX7-A $P=0.0013$, Scr-shPAX7-D $P=0.0009$ ) (SJRHB015721_X1 Scr-shPAX7-A $P=0.0406$, Scr-shPAX7-D P = 0.0092) (SJRHB011_Y1 ScrshPAX7-A $P<0.0001$, Scr-shPAX7-D $P=0.0318$ ). e Cell viability by Cell Titer Glo assay of RD, SMS-CTR, Rh18, SJRHB015721_X1, and SJRHB011_Y cell lines after $P A X 7$ knockdown, in quadruplicate. All $P$ values are $<0.0001$. $\mathbf{f}$ SMS-CTR proliferation assay following $P A X 7$ knockdown, $n=30$ images (six wells, five images taken per well). $\mathbf{g}$-i Tumor volume over time $(\mathbf{g}, P<0.0001)$ and final tumor mass $(\mathbf{h}, P=0.0003)$ of orthotopically injected SMS-CTR cells in SCID/Beige mice ( $n=10$ per group) transduced with PAX7 targeting or scramble shRNAs. $\mathbf{i}$ representative histology of tumors from $(\mathbf{g}), n=10$. $\mathbf{j}-\mathbf{I}$ Tumor volume over time (i, all $P$ values $<0.0001$ ) and final tumor mass (j, Scr-shPAX7-A $P=0.0028$, Scr-shPAX7-D $P=0.0006$ ) of orthotopically injected SJRHB015721_X1 cells in SCID/Beige mice transduced with PAX7 targeting or scramble shRNAs, $n=6$. I representative histology of tumors from (j), $n=6$. All $P$ values in pairwise comparisons were determined by Student's $t$ test (unpaired, two-tailed); ${ }^{\star} P<0.05,{ }^{\star \star} P<0.01,{ }^{\star \star \star} P<0.001,{ }^{\star \star \star \star \star} P<0.0001$. Data represented as mean \pm SEM. See also Supplementary Fig. 7. Source data are provided as a Source Data file.

PTEN/PAX7 loss in our Smoothened-driven tumors allows the outgrowth of a precursor lesion to become more fully manifested. Furthermore, since PAX7 depletion in established tumors did not cause a shift to smooth-muscle differentiation, our data suggest that these fate decisions are made early on in tumorigenesis and that tumor lineage factors like PAX7 become more 

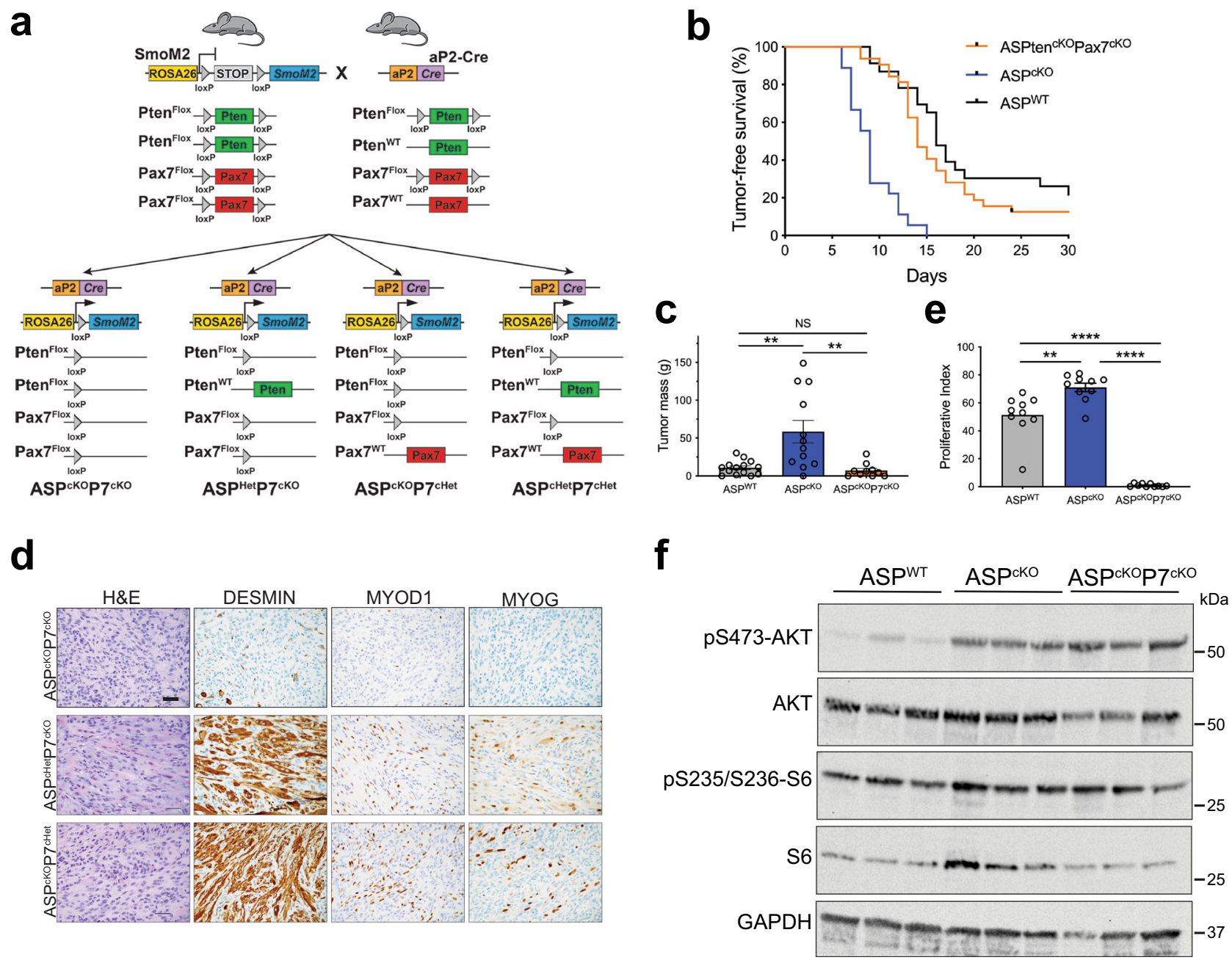

Fig. 7 PAX7 loss rescues deleterious effects of Pten loss in ASPcKo tumors. a Breeding strategy to generate ASPcKOP7cKO tumor mice. b Kaplan-Meier tumor-free survival curve of ASPCKOP7CKO $(n=32)$ in comparison to ASPWT and ASPcKO mice (from Fig. 1b). Mantel-Cox log rank, $P<0.0001$. c Tumor mass of ASPcKOP7CKO $(n=10)$, ASPWT $(n=11)$ and ASPcKO $(n=12)$ tumors at P12. ASPWT and ASPcKO data are the same from Fig. 1c. (ASPWT-ASPcKO $P=0.0022$, ASPCKO-ASPCKOP7CKO $P=0.0056)$. d Representative histology of ASPcKOP7cKO $(n=14)$, ASPcHetP7cKO $(n=4)$, and ASPcKOP7cHet $(n=4)$ tumors by H\&E staining and IHC for DESMIN, MYOD1, and MYOG. Scale bar, $25 \mu \mathrm{m}$. e Quantitation of percent Ki67-positive nuclei in ASPWT ( $n=9$ ), ASPCKO tumors $(n=7)$, and ASPCKOP7cKO $(n=5)$ tumors (ten random fields of view per genotype). ASPWT and ASPcKO from Fig. 1f. (ASPWT-ASPcKO $P=0.0029$, ASPWT-ASPCKOP7CKO $P<0.0001$, ASPCKO_ASPCKOP7CKO $P<0.0001$ ). f Immunoblots of phosphorylated AKTSer473, AKT, phosphorylated $\mathrm{S} 6 \mathrm{Ser235/236}, \mathrm{S6}$, and GAPDH, $n=3$. All $P$ values in pairwise comparisons were determined by Student's $t$ test (unpaired, two-tailed); NS not significant, ${ }^{\star \star} P<0.01,{ }^{\star \star \star \star} P<0.0001$. Data represented as mean \pm SEM. Source data are provided as a Source Data file.

focused on tumor maintenance after these fate decisions are made. This may explain why development-related tumors, such as pediatric tumors like RMS, are highly dependent on transcriptional regulators ${ }^{54}$. Although their loss early on in tumorigenesis may alter the ultimate tumor presentation, clinically, these tumors may have become more addicted to these factors in tumor maintenance; this may allow for therapeutic intervention of these tumors-either through indirect mechanisms (such as with HDAC inhibition in FP-RMS) or with directly-targeted molecular glues or protein degraders as these technologies improve.

The presented work details how the synthetic essential relationship dictates tumor fate in vivo. This synthetic essential relationship between Pten and Pax7 highlights the complex interplay between genetic alterations and transcriptional states that not only define tumorigenicity but even tumor origin and evolution. Taken together, this work illustrates how incredibly complex the interactions between the cell of origin, mutational spectrum, and transcriptional state are in dictating tumor lineage, and ultimately, therapeutic vulnerabilities.

\section{Methods}

Genetically engineered mouse models. All mouse strains are reported: $a P 2$ $\mathrm{Cre}^{22}$, R26-tdTomato (\#7914, The Jackson Laboratory (JAX) $)^{55}$, SmoM2 (\#5130, JAX) ${ }^{56}$, Pten $^{\text {flox }}(\# 6440, \text { JAX })^{57}$, Pax $^{\text {flox }}\left(\# 12653\right.$, JAX) ${ }^{58}$, Cdkn2a flox (Nabeel Bardeesy ${ }^{59}, \operatorname{Trp} 53^{f l o x}(\# 8462, \mathrm{JAX})^{60}$, and Rb1 flox (\#01XC1, US NCI) ${ }^{60}$. All mice were fed and watered ad libitum with consistent access to food and water, and all mice were housed in a facility kept at ambient temperature and humidity with $12-\mathrm{h}$ light/12-h dark cycles. Anterior necks lightly palpated while scruffed to feel for tumors and scored when first felt. All animal experiments were reviewed and approved by the SJCRH Institutional Animal Care and Use Committee.

Cell lines, primary cell culture, and patient-derived xenografts (PDXs). 293T (Martine Roussel, SJCRH), RD (\#CCL-136, ATCC), Rh18 (Children's Oncology Group Cell Line Repository, Monrovia, CA, USA), SMS-CTR (Rene Galindo, UTSW Medical Center), Rh36 (Christopher Morton, SJCRH), TE441 (\#CRL-7677, ATCC), Rh30 (\#CRL-2261, ATCC), and Rh2, Rh6, Rh3, Rh4, Rh28, and Rh41 (Gerard Grosveld, SJCRH) cells were maintained in DMEM (\#SH32043, HyClone) 
a

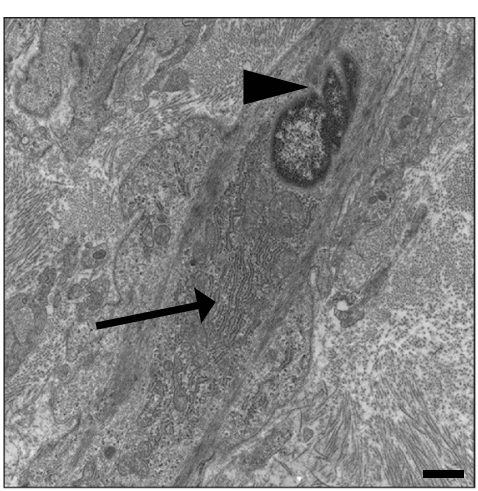

b
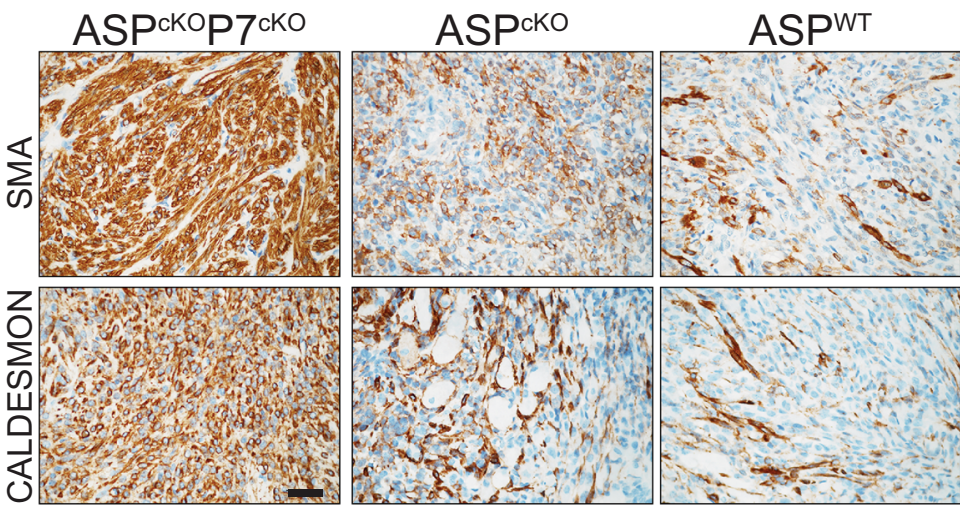

f

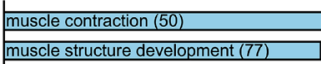

cell development (157)

organ morphogenesis (90)

muscle tissue morphoglenesis (21)

regulation of cell prolleration (112)

striated muscle cell development (27)

positive regulation of metabolic process (172)

regulation of celld dievelopment (75)

cell surface receptor signaling pathway (133)

$\begin{array}{llllll}0 & 5 & 10 & 15 & 20 & 25\end{array}$

$-\log _{10} p$ value

9

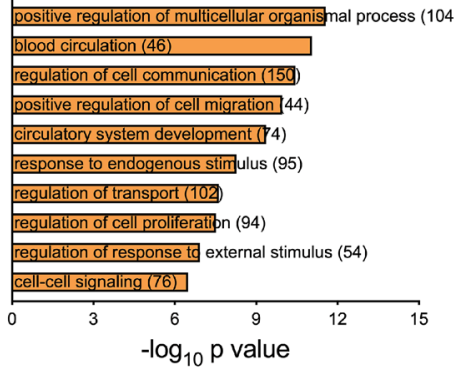

i

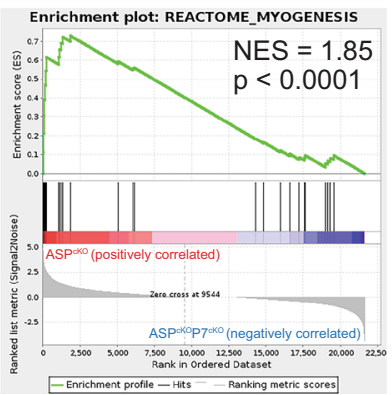

j

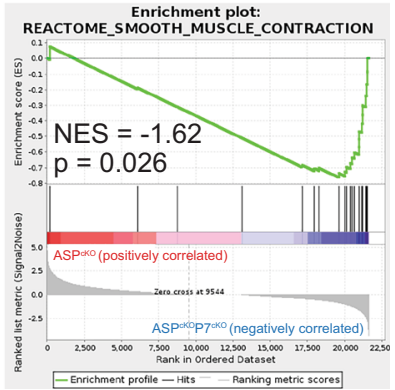

Fig. 8 PAX7 dictates rhabdomyosarcoma identity in ASPcKO tumors. a Representative transmission electron micrograph of an ASPcKOP7cKO tumor $(n=3)$ showing hallmarks of LMS; arrowhead = grooved nuclei, and arrow = prominent rough endoplasmic reticulum. Scale bar $=1 \mu \mathrm{m}$. $\mathbf{b}$ Representative histology of smooth-muscle differentiated tumor markers SMA and CALDESMON in ASPCKOP7CKO ( $n=14)$, ASPCKO $(n=4)$, and ASPWT ( $n=4)$ tumors. Scale bar, $25 \mu \mathrm{m}$. c, d Principal component analysis (c) and a volcano plot (d) of top 3000 differentially expressed genes (for $c$ ) and the significantly different $(P<0.05)$ and fourfold differentially expressed genes between the flow cytometry-sorted ASPcKO and ASPcKOP7cKO tumor cells $(\mathbf{d})$. Data represented as the log2 fold change of ASPCKO/ASPcKOP7cKO. Gold indicates the 1002 genes enriched in the ASPcKOP7cKO tumor cells. Blue indicates the 973 genes enriched in the ASPCKO tumor cells. Complete RNAseq data shown in Supplementary Data 3. $P$ values calculated by two-tailed unpaired $t$ test in limma package. e Heatmap of selected significantly $(P<0.05)$ differentially expressed genes ( $>$ fourfold, mean centered per gene) between tdTomato $+/$ CD31- tumor cell populations from ASTPCKO and ASTPCKOP7CKO tumors, $n=4$ tumors per genotype. $\mathbf{f}, \mathbf{g}$ GO analysis of differentially expressed genes (up in $\mathrm{ASPCKO},(\mathbf{f})$ ) (up in ASPcKOP7CKO, (g)) between flow cytometry-sorted tumor populations from both genotypes; top ten unique GO terms depicted following DAVID functional annotation clustering. h Normalized enrichment scores of gene sets enriched ASPcKO tumors compared to ASPcKOP7cKO tumors according to Gene Set Enrichment Analysis (GSEA). Dark blue = enriched in ASPcKO and light blue =enriched in ASPcKOP7cKO. $\mathbf{i}, \mathbf{j}$ Enrichment plots of skeletal myogenic (i, Reactome, myogenesis) and smooth muscle (j, Reactome, smooth-muscle contraction) gene signatures from (h). See also Supplementary Fig. 8 and Supplementary Data 3. Source data are provided as a Source Data file. 
supplemented with $10 \%$ fetal bovine serum (Hyclone) and $1 \%$ antibiotic/antimycotic (\#A5955, Sigma). SJRHB015721_X1 and SJRHB011_Y PDX-derived cell lines were harvested from SCID/Beige hindlimbs, dissociated, and plated in complete neurobasal media as described ${ }^{20,24}$. Briefly, tumors were digested by mechanical and enzymatic digestion with a buffered solution of collagenase II and trypsin for $1 \mathrm{~h}$ at $37^{\circ} \mathrm{C}$. Digested tumors were then filtered into single-cell suspensions and then plated into tissue-culture-treated plates. Primary rhabdospheres from ASPcKO tumors and PDXs were dissociated similarly as above, except in the case of the primary mouse tumors the enzymatic digestion buffer contained collagenase B and dispase II; tumor or PDX cells were plated in low-attachment plates after sterile filtering into single-cell suspensions. Cell lines authenticated by short tandem-repeat profiling and monitored for mycoplasma contamination (Universal Mycoplasma Detection Kit, ATCC, \#30-1012 K). Human skeletal muscle myoblasts obtained from Lonza (\#CC-2580) and grown according to the manufacturer's instructions. Mouse embryonic fibroblasts (MEFs) were harvested from E11.5 to 13.5 embryos. Wild-type MEFs immortalized by retroviral transduction with large $\mathrm{T}$ (genomic) antigen (\#1778, Addgene, a gift from Bob Weinberg ${ }^{61}$ ). All cell lines and rhabdospheres were maintained in a humidified incubator at constant $37^{\circ} \mathrm{C}$ and $5 \% \mathrm{CO}_{2}$.

RMS PDX samples (SJRHB010468_X1, SJRHB010463_X16, SJRHB010927_X1, SJRHB010928_X1, SJRHB013757_X2, SJRHB012405_X1, SJRHB013758_X1, SJRHB013759_X1, SJRHB013758_X2, SJRHB015721_X1, SJRHB15720_X1, SJRHB011_X, SJRHB011_Y, SJRHB012 X, SJRHB012 Y, SJRHB012 Z,

SJRHB013_X, and SJRHB000026_X1) were obtained from the Children's Solid Tumor Network (CSTN $)^{31}$. The CSTN serves as a repository for deidentified PDXs developed under the MAST (Molecular Analysis of Solid Tumors) protocol from NCT01050296 ${ }^{62}$. NCT01050296 was approved by St. Jude Children's Research Hospital's Institutional Review Board. Informed consent was obtained from each trial participant prior to be enrolled in the MAST clinical trial.

Histology and immunostaining. Fresh frozen or formalin-fixed paraffin-embedded tumor tissues were prepped for immunohistochemistry as described ${ }^{20}$. Briefly, paraffin-embedded tissues were rehydrated followed by antigen-retrieval (conditions listed in Supplementary Table 1). Frozen tissue sections were fixed in $4 \%$ paraformaldehyde overnight and cryoprotected in $30 \%$ sucrose, $2 \mathrm{mM} \mathrm{MgCl}$. Fixed tumor tissue was flash-frozen in liquid-nitrogen-cooled 2-methyl-butane and sectioned on a conventional cryostat. Hematoxylin \& eosin (H\&E) plus immunohistochemistry were performed using standard staining procedures. Antibodies are listed in Supplementary Table 2. Images captured on a Nikon Eclipse 80i upright fluorescent microscope or a Leica DMi 8 Thunder Imager inverted fluorescent microscope; images analyzed with either Nikon Elements Basic Research (v4.1.3) or the Leica Application Suite X v3.7.2 software. For PAX7 immunofluorescence, signal amplification with the TSA-Plus Cyanine 5 (\#NEL745001KT, PerkinElmer) performed according to the manufacturer's instructions. Images quantified with Nikon Elements Basic Research (v4.1.3) or FIJI's (ImageJ, NIH) total nuclei cell counter. Specimens for transmission electron micrographs prepared and imaged as described in ${ }^{41}$. Briefly, tumor tissue was fixed in $2.5 \%$ glutaraldehyde, $2 \%$ paraformaldehyde, in $0.1 \mathrm{M}$ sodium cacodylate buffer (pH 7.4) and post-fixed in $2 \%$ osmium tetroxide in $0.1 \mathrm{M}$ cacodylate buffer with $0.15 \%$ potassium ferrocyanide. Samples were dehydrated in increasing ethanol concentration washes to propylene oxide. Samples embedded overnight at $70^{\circ} \mathrm{C}$ in epoxy resin; ultrathin $(80 \mathrm{~nm})$ sections were imaged on a JEOL 1200EX TEM with an AMT XR111 or Tecnai TF20 TEM with an AMT XR41 camera.

Fluorescence-activated cell sorting. ASPcKO and ASPcKOP7 ${ }^{\mathrm{CKO}}$ tumors dissected and manually dissociated, digested, and stained for FACS prior to analysis with a FACS Aria Cellsorter (BD Biosciences) as described ${ }^{20}$. Briefly, tumors were mechanically and enzymatically digested in a buffered solution of collagenase $B$ and dispase II for $1 \mathrm{~h}$ at $37^{\circ} \mathrm{C}$; tumor cells were then sterile filtered to single-cell suspensions and stained for 30 min with 1:50 anti-CD31-APC prior to flow cytometry. Antibody information is detailed in Supplementary Table 2. DAPI was used for live/dead cell marker.

Viability assays and pharmacological agents. Cells were assessed for viability with CellTiterGlo (\#G7570, Promega) or periodic image acquisition with confluence calculation (Incucyte S3, Essen BioScience). CellTiterGlo assays were performed according to the manufacturer's protocol; luminescence was measured on a BioTek Synergy 2 and BioTek's Gen5 1.11 software. Doxorubicin (\#D-4000) and everolimus (\#E-4040) were from LC Labs. GDC-0941 (\#S1065), trametinib (\#S2673) and MK-2206 (\#S1078) were from Selleck Chemicals.

Molecular cloning, viral transduction, and luciferase assays. Gene blocks (Integrated DNA Technologies (IDT), Coralville, IA, USA) designed for the murine Pax7 mRNA coding sequence were cloned into the pBABE-puro retroviral vector (Hartmut Land, Jay Morgenstern, and Bob Weinberg, \#1764, Addgene). Retroviruses packaged with pCL-Eco (Inder Varma, \#12371, Addgene) and transfected into 293T cells. MEFs were transduced with conditioned 293T media containing viral particles after filtering $(0.22 \mu \mathrm{m})^{63}$.
Lentiviruses encoding shRNAs (pLKO.1 or pLKO.3 G vectors, Bob Weinberg, \#8453, Addgene ${ }^{64}$, or Christophe Benoist and Diane Mathis, \#14748, Addgene) packaged and transfected into $293 \mathrm{~T}$ cells, and adherent cells were transduced as the MEFs above ${ }^{65}$. For primary murine rhabdosphere transduction, virus was filtered and incubated overnight at $4^{\circ} \mathrm{C}$ in one-third volume of Lenti-X (\#631232, Takara). Concentrated viral particles in 1-2 mL of complete neurobasal medium and plated in ultra-low-attachment plates for overnight transduction at $37^{\circ} \mathrm{C}$. For primary human PDX transduction, the filtered virus was added to pelleted PDX cells and incubated for $2.5 \mathrm{~h}$ at $37^{\circ} \mathrm{C}$.

MEFs infected with adenovirus-Cre recombinase (VVC-U of Iowa-1174) or GFP (VVC-U of Iowa-4) (University of Iowa Viral Vector Core) overnight (1 $\mu \mathrm{g}$ virus/ $1 \mathrm{~mL}$ medium).

The 900 bp cis-regulatory region immediately prior to the $D b x 1$ transcription start site was synthesized (gene block, IDT) and subcloned into pGL4.25[luc2CP/ $\min P]$ (\#E8431, Promega). Synthesized Pax7 cDNA was cloned into pCMV6 (Eric Olson). 293Ts were co-transfected with $280 \mathrm{ng}$ pGL4.25-Dbx1 promoter, $20 \mathrm{ng}$ pCMV6-LacZ (as transfection control), and dose-dependent increases in Pax7 cDNA. FuGene (\#E2692, Promega) was transfection reagent. Luciferase activity was measured using the Promega Luciferase Assay System (Promega, E1501), and fluorescence activity was measured using the LacZ/Galactosidase Quantification Kit (FluoReporter, ThermoScientific, F-2905); both measurements were made on a BioTek Synergy 2 . Luciferase activity was normalized to $\beta$-galactosidase activity relative to control ${ }^{65}$.

Chromatin immunoprecipitation. Low-passage SJRHB015721_X1-adherent cells were grown to $\sim 80 \%$ confluency; cells were collected in PBS by scraping. Cells were cross-linked in $1.1 \%$ formaldehyde in PBS, quenched with $125 \mathrm{mM}$ glycine, and lysed in ChIP lysis buffer $3[10 \mathrm{mM}$ Tris- $\mathrm{HCl}(\mathrm{pH} 8.0), 100 \mathrm{mM} \mathrm{NaCl}, 1 \mathrm{mM}$ EDTA, $0.5 \mathrm{mM}$ EGTA, $0.1 \%$ sodium deoxycholate, $0.5 \%$ N-lauroylsarcosine] with $1 \times$ protease inhibitors, $1 \mathrm{mM}$ DTT, and $1 \mathrm{mM}$ PMSF. The fixed and lysed cells were sonicated with a Covaris M220 (Covaris) sonicator with the following conditions: peak incident power-75, duty factor $-15 \%$, cycles per burst-200, temperature $-7^{\circ} \mathrm{C}$ for $12 \mathrm{~min}$ in $1 \mathrm{~mL}$ lysis buffer. Chromatin was incubated with prewashed protein A/G Dynabeads (Life Technologies) and $4 \mu \mathrm{g}$ PAX7 antibody (DSHB) overnight at $4{ }^{\circ} \mathrm{C}$. After incubation, beads were washed and immunoprecipitates were eluted. DNA from eluates was isolated using standard phenol:chloroform extraction and resuspended in low-EDTA TE buffer. Supplementary Table 1 lists the ChIP-qPCR primers used. ChIP-qPCR signals were calculated as fold enrichment over IgG control antibody.

Spectral karyotyping. ASP ${ }^{c K O}$ or ASP WT tumors were analyzed for genomic alterations. Mice were intraperitoneally injected with $400 \mu \mathrm{L}$ of $10 \mu \mathrm{g} / \mathrm{mL}$ colcemid and tumors were harvested $4 \mathrm{~h}$ post injection. Tumor cells were cultured in RPMI1640 (supplemented with $10 \%$ fetal bovine serum and $1 \%$ penicillin/streptomycin) and harvested up to 10 days post culture using routine cytogenetic methods. A commercially prepared SKY probe from Applied Spectral Imaging (Carlsbad, CA USA) was used as a probe for this analysis and manufacturer protocols were used for hybridization and detection steps. Two mice $(n=68$ metaphase cells per genotype) from each genotype were analyzed.

Immunoblotting and reverse-phase protein array (RPPA). Immunoblotting was performed with standard procedures. Antibodies are listed in Supplementary Table 2. For RPPA, protein lysates were prepared according to protocols provided by the MD Anderson Cancer Center Functional Proteomics RPPA Core (supported by MD Anderson Cancer Center Support Grant \#5 P30 CA016672-40). The RPPA core performed the array and analyzed the data. $\log _{2}$-transformed data, mediancentered, were used to generate the heatmap.

In vivo tumorigenesis. Murine tumors dissected into $\sim 27-\mathrm{mm}^{3}$ cubes and placed into serum-free Media 199 (\#11150-067, Gibco) supplemented with 1\% antibiotic/ antimycotic (\#A5955, Sigma) prior to implantation into SCID/Beige (\#250, Charles River Laboratories) or CB17 (\#CB17SC, Taconic Biosciences) mice. Allografts implanted via flank incision and closed with VetBond (\#14695B, $3 \mathrm{M}$ ).

Dissociated PDX samples were transduced with lentivirus for $2.5 \mathrm{~h}$. One million PDX cells/mouse were resuspended in $100 \mu \mathrm{L}$ matrigel (\#354234, Corning) and implanted into the left hindlimb of adult SCID/Beige mice. Volumes calculated as $\pi / 6 \times[(\mathrm{L}+\mathrm{W}) / 2] 3^{63}$; tumor volume reported as the difference between the injected and contralateral, uninjected limb.

RNA isolation and gene expression analysis. RNA was isolated using a Qiagen miRNeasy Mini (\#217004, Valencia, CA, USA) or Micro (\#217084) (for sorted tumor populations and primary tumorsphere experiments) kit according to the manufacturer instructions. Reverse transcription was performed with Superscript III First Strand cDNA Synthesis Kit using random hexamer primers (ThermoFisher, \#180800551). SYBR primers and Taqman probes used are listed in Supplementary Table 1 . Data normalized to Actb/ACTB or $18 \mathrm{~S}$ rRNA expression. 
For microarray analysis changes between ASPcKO and ASPWT tumors, amplified cDNA was prepared using an Ovation Pico WTA System V2 (\#3302, Nugen, San Carlos, CA) and analysis was performed on a Mouse 2.0 ST microarray (\#902118, Affymetrix, Santa Clara, CA, USA $)^{20}$. Data were imported into Partek Genome Suite 6.6, visualized by principal component analysis, batch corrected, and statistically tested using an unequal variance $t$ test. Unannotated transcripts were filtered out and imported into STATA/MP 14.2 and $P$ values adjusted for multiple comparisons by the Benjamini-Hochberg FDR method ${ }^{66}$. Volcano plots were also produced in STATA/MP 14.2 by plotting the $\log _{10}$ transformed $P$ value from the unequal variance $t$ test against the log ratio of expression.

CIBERSORT analysis for predicted differences in immune cell compartments within ASPWT and ASPcKO tumors was performed with the LM22 leukocyte gene signature ${ }^{67}$

Gene expression changes between shDBX1-transduced and shScrambledtransduced SJRHB015271 X1 cells, Clariom S human microarrays were used. In total, 125 ng RNA was processed using ThermoFisher (Affymetrix) Whole Transcript (WT) Plus assay kit (\#902280). Labeled cDNA included in a hybridization mix incubated on the Clariom $\mathrm{S}$ human array for $16 \mathrm{~h}$ at $45^{\circ} \mathrm{C}$ while rotating at $60 \mathrm{rpm}$. Cartridges were stained and washed on the Gene Chip FS450 fluidics station and scanned on the Gene Chip Scanner 3000 7G. Data were imported, visualized by principal component analysis, statistically analyzed, and rendered as described above.

Low-input RNA sequencing was performed for gene expression changes between flow cytometry-sorted tumor populations from the ASPcKOP7 ${ }^{\mathrm{CKO}}$ and ASPcKO tumors. In all, 5 ng RNA was used in the Tecan Ovation RNA sequencing system v2 protocol as written to create SPIA (single primer isothermal amplification)-cDNA. Once purified, $500 \mathrm{ng}$ of the sample was sheared (Covaris LE220 focused ultra-sonicator (96 microtube-50 AFA fiber plate, target base pair size of 300; shearing settings: Peak Incident Power (W) of 450 , duty factor of $15 \%$, cycles per burst 1000 with a $100 \mathrm{~s}$ treatment time)). Libraries were created with sheared cDNA and the KAPA Hyper-Prep kit (\#KK8604, Roche). Four PCR cycles used for the cDNA amplification step and the protocol was followed according to the manufacturer's instructions. UDI DNA indexes from Illumina (\#20022370) were used.

Total stranded RNA sequencing data were processed by the internal AutoMapper pipeline. Raw reads were first trimmed (Trim-Galore version 0.60 ), mapped to mouse genome assembly (GRCm38) (STAR v2.7) ${ }^{68}$ and then the gene level values were quantified (RSEM v1.31) based on GENCODE annotation (vM22). Low count genes removed from analysis using a CPM cutoff corresponding to a count of ten reads and only confidently annotated (level 1 and 2 gene annotation) and protein-coding genes are used for differential expression analysis. Normalization factors were generated using the TMM method; counts then transformed using voom. Transformed counts analyzed using the $\operatorname{lmFit}$ and eBayes functions (R limma package version 3.42.2). The significantly up- and downregulated genes were defined by at least fold change $>4$ and $P$ value $<0.05$.

For gene ontology (GO) analyses, Gene Set Enrichment Analysis (GSEA) or functional annotation clustering using the Database for Annotation, Visualization, and Integrated Discovery (DAVID, version 6.8) was used. For GSEA, the GSEA software ${ }^{69}$ version 4.0 was used and based on the pre-ranked option. Normalized enrichment score used for visualization. For DAVID ${ }^{70}$, functional annotation clustering using the GOTERM_BP_FAT selection was performed. Top ten unique GO terms visualized and represented as the $-\log _{10} P$ value.

Statistics. Sample size and replicates are listed in Figure legends. Error bars reported as mean \pm SEM and statistical significance determined by using two-tailed, unpaired Student's $t$ test for pairwise comparisons unless otherwise indicated. Kaplan-Meier survival analyses were performed by log-rank (Mantel-Cox) tests. $P$ values below 0.05 considered significant and indicated by the following: ${ }^{*} P<0.05$, ${ }^{* *} P<0.01,{ }^{* * *} P<0.001,{ }^{* * *} P<0.0001$. GraphPad Prism 9 was used for statistical tests. Statistical analyses of gene expression data described above.

Reporting summary. Further information on research design is available in the Nature Research Reporting Summary linked to this article.

\section{Data availability}

Microarray and RNAseq data generated are deposited in the Gene Expression Omnibus (GSE166906). St. Jude ProteinPaint RNA sequencing data accessed through their openresource page (https://pecan.stjude.cloud) ${ }^{29}$. RNA sequencing data from the US National Cancer Institute RMS dataset accessed from ${ }^{30}$. RNA sequencing and dependency data (CERES score $)^{28}$ from the Broad Institute's Cancer Dependency Map accessed from the 20Q3 data deposit (https://depmap.org/portal/). St. Jude and US NCI RMS coreregulatory circuit data are previously published and referenced, respectively ${ }^{34,35}$. Interspecies sequence conservation for the $D B X 1$ promoter was performed using the Evolutionary Conserved Regions database (https://ecrbrowser.dcode.org) ${ }^{71}$. Transcription factor binding sites found within the murine $D b x 1$ promoter were mined from the JASPAR 2016 database (https://jaspar2016.genereg.net) ${ }^{72}$. All other relevant data supporting the key findings of this study are available within the article and its Supplementary Information files, Source Data file, or from the corresponding author,
Mark E. Hatley (mark.hatley@stjude.org), upon reasonable request. A Reporting Summary for this article is available in a Supplementary Information file. Source data are provided with this paper.

Received: 5 April 2021; Accepted: 1 September 2021; Published online: 17 September 2021

\section{References}

1. Kashi, V. P., Hatley, M. E. \& Galindo, R. L. Probing for a deeper understanding of rhabdomyosarcoma: insights from complementary model systems. Nat. Rev. Cancer 15, 426-439 (2015).

2. Skapek, S. X. et al. Rhabdomyosarcoma. Nat. Rev. Dis. Prim. 5, 1 (2019).

3. Chen, $X$. et al. Targeting oxidative stress in embryonal rhabdomyosarcoma. Cancer Cell 24, 710-724 (2013).

4. Paulson, V. et al. High-resolution array CGH identifies common mechanisms that drive embryonal rhabdomyosarcoma pathogenesis. Genes, Chromosomes Cancer 50, 397-408 (2011).

5. Shern, J. F. et al. Comprehensive genomic analysis of rhabdomyosarcoma reveals a landscape of alterations affecting a common genetic axis in fusionpositive and fusion-negative tumors. Cancer Discov. 4, 216-231 (2014).

6. Seki, M. et al. Integrated genetic and epigenetic analysis defines novel molecular subgroups in rhabdomyosarcoma. Nat. Commun. 6, 7557 (2015).

7. Shukla, N. et al. Oncogene mutation profiling of pediatric solid tumors reveals significant subsets of embryonal rhabdomyosarcoma and neuroblastoma with mutated genes in growth signaling pathways. Clin. Cancer Res. 18, 748-757 (2012).

8. Yuan, T. L. \& Cantley, L. C. PI3K pathway alterations in cancer: variations on a theme. Oncogene 27, 5497-5510 (2008).

9. Jiang, B. H. \& Liu, L. Z. PI3K/PTEN signaling in angiogenesis and tumorigenesis. Adv. Cancer Res 102, 19-65 (2009).

10. Shern, J. F. et al. Genomic classification and clinical outcome in rhabdomyosarcoma: a report from an International Consortium. J. Clin. Oncol. https://doi.org/10.1200/jco.20.03060 (2021).

11. Lee, Y. R., Chen, M. \& Pandolfi, P. P. The functions and regulation of the PTEN tumour suppressor: new modes and prospects. Nat. Rev. Mol. Cell Biol. 19, 547-562 (2018)

12. Steinbach, N. et al. PTEN interacts with the transcription machinery on chromatin and regulates RNA polymerase II-mediated transcription. Nucleic Acids Res. 47, 5573-5586 (2019).

13. Cho, H. et al. RapidCaP, a novel GEM model for metastatic prostate cancer analysis and therapy, reveals myc as a driver of Pten-mutant metastasis. Cancer Discov. 4, 318-333 (2014).

14. Granados, V. A. et al. Selective targeting of myoblast fusogenic signaling and differentiation-arrest antagonizes rhabdomyosarcoma cells. Cancer Res. 79, 4585-4591 (2019).

15. Manzella, G. et al. Phenotypic profiling with a living biobank of primary rhabdomyosarcoma unravels disease heterogeneity and AKT sensitivity. Nat. Commun. 11, 4629 (2020).

16. McKinnon, T. et al. Functional screening of FGFR4-driven tumorigenesis identifies $\mathrm{PI} 3 \mathrm{~K} / \mathrm{mTOR}$ inhibition as a therapeutic strategy in rhabdomyosarcoma. Oncogene 37, 2630-2644 (2018).

17. Zhao, D. et al. Synthetic essentiality of chromatin remodelling factor CHD1 in PTEN-deficient cancer. Nature 542, 484-488 (2017).

18. Chatterjee, N. et al. Synthetic essentiality of metabolic regulator PDHK1 in PTEN-deficient cells and cancers. Cell Rep. 28, 2317-2330.e2318 (2019).

19. $\mathrm{Xu}$, L. et al. Integrative Bayesian analysis identifies rhabdomyosarcoma disease genes. Cell Rep. 24, 238-251 (2018).

20. Drummond, C. J. et al. Hedgehog pathway drives fusion-negative rhabdomyosarcoma initiated from non-myogenic endothelial progenitors. Cancer Cell 33, 108-124 e105 (2018).

21. Hatley, M. E. et al. A mouse model of rhabdomyosarcoma originating from the adipocyte lineage. Cancer Cell 22, 536-546 (2012).

22. Tang, W. et al. White fat progenitor cells reside in the adipose vasculature. Science 322, 583-586 (2008)

23. Chal, J. \& Pourquie, O. Making muscle: skeletal myogenesis in vivo and in vitro. Development 144, 2104-2122 (2017).

24. Walter, D. et al. CD133 positive embryonal rhabdomyosarcoma stem-like cell population is enriched in rhabdospheres. PLOS ONE 6, e19506 (2011).

25. Pierani, A. et al. Control of interneuron fate in the developing spinal cord by the progenitor homeodomain protein Dbx1. Neuron 29, 367-384 (2001).

26. Karaz, S. et al. Neuronal fate specification by the Dbxl transcription factor is linked to the evolutionary acquisition of a novel functional domain. Evodevo 7, 18 (2016).

27. Champagnat, J., Morin-Surun, M. P., Bouvier, J., Thoby-Brisson, M. \& Fortin, G. Prenatal development of central rhythm generation. Respir. Physiol. Neurobiol. 178, 146-155 (2011). 
28. Tsherniak, A. et al. Defining a cancer dependency map. Cell 170, 564-576 e516 (2017).

29. Zhou, X. et al. Exploring genomic alteration in pediatric cancer using ProteinPaint. Nat. Genet. 48, 4-6 (2016).

30. Gryder, B. E. et al. PAX3-FOXO1 establishes myogenic super enhancers and confers BET bromodomain vulnerability. Cancer Discov. 7, 884-899 (2017).

31. Stewart, E. et al. The childhood solid tumor network: a new resource for the developmental biology and oncology research communities. Dev. Biol. 411, 287-293 (2016).

32. Hanna, J. A. et al. PAX7 is a required target for microRNA-206-induced differentiation of fusion-negative rhabdomyosarcoma. Cell Death Dis. 7, e2256 (2016).

33. von Maltzahn, J., Jones, A. E., Parks, R. J. \& Rudnicki, M. A. Pax7 is critical for the normal function of satellite cells in adult skeletal muscle. Proc. Natl Acad. Sci. USA 110, 16474-16479 (2013).

34. Stewart, E. et al. Identification of therapeutic targets in rhabdomyosarcoma through integrated genomic, epigenomic, and proteomic analyses. Cancer Cell 34, 411-426 e419 (2018).

35. Gryder, B. E. et al. Histone hyperacetylation disrupts core gene regulatory architecture in rhabdomyosarcoma. Nat. Genet. 51, 1714-1722 (2019).

36. Seale, P. et al. Pax7 is required for the specification of myogenic satellite cells. Cell 102, 777-786 (2000).

37. Basch, M. L., Bronner-Fraser, M. \& Garcia-Castro, M. I. Specification of the neural crest occurs during gastrulation and requires Pax7. Nature 441, 218-222 (2006).

38. Wang, Z., Wang, D. Z., Pipes, G. C. \& Olson, E. N. Myocardin is a master regulator of smooth muscle gene expression. Proc. Natl Acad. Sci. USA 100, 7129-7134 (2003).

39. Guo, X. et al. Clinically relevant molecular subtypes in leiomyosarcoma. Clin. Cancer Res. 21, 3501-3511 (2015).

40. Lee, K. Y. et al. Lessons on conditional gene targeting in mouse adipose tissue. Diabetes 62, 864-874 (2013).

41. Hanna, J. A. et al. Biallelic Dicer1 loss mediated by aP2-Cre drives angiosarcoma. Cancer Res. 77, 6109-6118 (2017).

42. Shintaku, M. \& Sekiyama, K. Leiomyosarcoma of the uterus with focal rhabdomyosarcomatous differentiation. Int. J. Gynecol. Pathol. 23, 188-192 (2004).

43. Verma, M., Joseph, G. \& McCluggage, W. G. Uterine composite tumor composed of leiomyosarcoma and embryonal rhabdomyosarcoma with immature cartilage. Int. J. Gynecol. Pathol. 28, 338-342 (2009).

44. Budry, L. et al. The selector gene Pax7 dictates alternate pituitary cell fates through its pioneer action on chromatin remodeling. Genes Dev. 26, 2299-2310 (2012).

45. Betters, E., Liu, Y., Kjaeldgaard, A., Sundström, E. \& García-Castro, M. I. Analysis of early human neural crest development. Dev. Biol. 344, 578-592 (2010).

46. Lee, Q. Y. et al. Pro-neuronal activity of Myod1 due to promiscuous binding to neuronal genes. Nat. Cell Biol. 22, 401-411 (2020).

47. Galli, R. et al. Skeletal myogenic potential of human and mouse neural stem cells. Nat. Neurosci. 3, 986-991 (2000).

48. Duan, S. et al. PTEN deficiency reprogrammes human neural stem cells towards a glioblastoma stem cell-like phenotype. Nat. Commun. 6, 10068 (2015).

49. Gryder, B. E. et al. Chemical genomics reveals histone deacetylases are required for core regulatory transcription. Nat. Commun. 10, 3004 (2019).

50. Peng, W. et al. Loss of PTEN promotes resistance to T cell-mediated immunotherapy. Cancer Discov. 6, 202-216 (2016).

51. Zhao, D. et al. Chromatin regulator CHD1 remodels the immunosuppressive tumor microenvironment in PTEN-deficient prostate cancer. Cancer Discov. 10, 1374-1387 (2020).

52. Chen, P. et al. Symbiotic macrophage-glioma cell interactions reveal synthetic lethality in PTEN-Null glioma. Cancer Cell 35, 868-884.e866 (2019).

53. Ho, J., Cruise, E. S., Dowling, R. J. O. \& Stambolic, V. PTEN nuclear functions. Cold Spring Harb. Perspect. Med. 10, https://doi.org/10.1101/ cshperspect.a036079 (2020).

54. Dharia, N. V. et al. A first-generation pediatric cancer dependency map. Nat. Genet. 53, 529-538 (2021).

55. Madisen, L. et al. A robust and high-throughput Cre reporting and characterization system for the whole mouse brain. Nat. Neurosci. 13, 133-140 (2010).

56. Mao, J. et al. A novel somatic mouse model to survey tumorigenic potential applied to the Hedgehog pathway. Cancer Res. 66, 10171-10178 (2006)

57. Lesche, R. et al. Cre/loxP-mediated inactivation of the murine Pten tumor suppressor gene. Genesis 32, 148-149 (2002).

58. Lepper, C., Conway, S. J. \& Fan, C. M. Adult satellite cells and embryonic muscle progenitors have distinct genetic requirements. Nature 460, 627-631 (2009).

59. Aguirre, A. J. et al. Activated Kras and Ink4a/Arf deficiency cooperate to produce metastatic pancreatic ductal adenocarcinoma. Genes Dev. 17, 3112-3126 (2003).

60. Marino, S., Vooijs, M. van Der Gulden, H., Jonkers, J. \& Berns, A. Induction of medulloblastomas in p53-null mutant mice by somatic inactivation of $\mathrm{Rb}$ in the external granular layer cells of the cerebellum. Genes Dev. 14, 994-1004 (2000).
61. Hahn, W. C. et al. Enumeration of the simian virus 40 early region elements necessary for human cell transformation. Mol. Cell Biol. 22, 2111-2123 (2002)

62. Stewart, E. et al. Orthotopic patient-derived xenografts of paediatric solid tumours. Nature 549, 96-100 (2017).

63. Hatley, M. E. et al. Modulation of K-Ras-dependent lung tumorigenesis by MicroRNA-21. Cancer Cell 18, 282-293 (2010).

64. Stewart, S. A. et al. Lentivirus-delivered stable gene silencing by RNAi in primary cells. RNA 9, 493-501 (2003).

65. Hanna, J. A. et al. PAX3-FOXO1 drives miR-486-5p and represses miR-221 contributing to pathogenesis of alveolar rhabdomyosarcoma. Oncogene 37, 1991-2007 (2018).

66. Benjamini, Y. \& Hochberg, Y. Controlling the false discovery rate-a practical and powerful approach to multiple testing. J. R. Stat. Soc. B 57, 289-300 (1995)

67. Newman, A. M. et al. Robust enumeration of cell subsets from tissue expression profiles. Nat. Methods 12, 453-457 (2015).

68. Dobin, A. et al. STAR: ultrafast universal RNA-seq aligner. Bioinformatics 29, 15-21 (2013)

69. Subramanian, A. et al. Gene set enrichment analysis: a knowledge-based approach for interpreting genome-wide expression profiles. Proc. Natl Acad. Sci. USA 102, 15545-15550 (2005).

70. Huang da, W., Sherman, B. T. \& Lempicki, R. A. Systematic and integrative analysis of large gene lists using DAVID bioinformatics resources. Nat. Protoc. 4, 44-57 (2009)

71. Ovcharenko, I., Nobrega, M. A., Loots, G. G. \& Stubbs, L. ECR Browser: a tool for visualizing and accessing data from comparisons of multiple vertebrate genomes. Nucleic Acids Res. 32, W280-W286 (2004).

72. Mathelier, A. et al. JASPAR 2016: a major expansion and update of the openaccess database of transcription factor binding profiles. Nucleic Acids Res. 44, D110-D115 (2016)

73. Fornes, O. et al. JASPAR 2020: update of the open-access database of transcription factor binding profiles. Nucleic Acids Res. 48, D87-d92 (2020).

\section{Acknowledgements}

We thank the SJCRH Shared Resources including Flow Cytometry and Cell Sorting (Richard Ashmun), Veterinary Pathology Core (Peter Vogel, Pam Johnson), Cell and Tissue Imaging Electron Microscopy Services (Cam Robinson), the Core Cytogenetic Laboratory (Marc Valentine, Virginia Valentine), the Hartwell Center and Functional Genomics (Geoff Neale, Melanie Loyd), and the Center for Applied Bioinformatics (Gang Wu). We thank Johnathan Dallman, Jonathan Go, D. Wood Kimbrough, and Lauren Goudie for technical assistance. Research reported in this publication supported by the National Cancer Institute of the National Institutes of Health under Award Numbers R01CA216344 and R01CA251436 (MEH) and F31CA250398 (MB). The content is solely the responsibility of the authors and does not necessarily represent the official views of the National Institutes of Health. The Hatley laboratory is also supported by grants from The V Foundation for Cancer Research, the Rally Foundation for Childhood Cancer Research and Open Hands Overflowing Hearts award number 20IC23 (MEH), St. Jude Cancer Center Support Grant (P30 CA21765), and American Lebanese Syrian Associated Charities of St. Jude Children's Research Hospital. K.E.G. is supported by a Damon Runyon-Sohn Pediatric Cancer Fellowship (DRSG-30-19).

\section{Author contributions}

Conceptualization-C.G.L. and M.E.H.; methodology-C.G.L., M.R.G., K.E.G., M.K.E., J.A.H., and M.E.H.; formal analysis-C.G.L., J.E.R., and M.E.H.; investigation-C.G.L., K.E.G., M.R.G., M.K.E., K.B.R., M.B., J.A.H., C.J.D., P.A.S., P.J.L., and M.C.M.; data curation-D.F. and H.J.; writing: original draft-C.G.L. and M.E.H.; writing: reviewing and editing-all authors; visualization-C.G.L. and M.E.H.; supervision-M.E.H.; funding acquisition-M.E.H

\section{Competing interests}

The authors declare no competing interests.

\section{Additional information}

Supplementary information The online version contains supplementary material available at https://doi.org/10.1038/s41467-021-25829-4.

Correspondence and requests for materials should be addressed to Mark E. Hatley.

Peer review information Nature Communications thanks Charis Eng and the other, anonymous, reviewer(s) for their contribution to the peer review of this work.

Reprints and permission information is available at http://www.nature.com/reprints

Publisher's note Springer Nature remains neutral with regard to jurisdictional claims in published maps and institutional affiliations. 
(c) (i) Open Access This article is licensed under a Creative Commons Attribution 4.0 International License, which permits use, sharing, adaptation, distribution and reproduction in any medium or format, as long as you give appropriate credit to the original author(s) and the source, provide a link to the Creative Commons license, and indicate if changes were made. The images or other third party material in this article are included in the article's Creative Commons license, unless indicated otherwise in a credit line to the material. If material is not included in the article's Creative Commons license and your intended use is not permitted by statutory regulation or exceeds the permitted use, you will need to obtain permission directly from the copyright holder. To view a copy of this license, visit http://creativecommons.org/ licenses/by/4.0/.

(C) The Author(s) 2021 